verschiedene Urteile des EuGH, in denen er für Richtlinien, die die Regelung eines Privatrechtsverhältnisses zum Gegenstand haben, nur auf die Möglichkeit richtlinienkonformer Auslegung verwies, nicht aber auf die Ausschlusswirkung des Gemeinschaftsrechts. ${ }^{55}$ Legt man dieses Verständnis der Rechtsprechung zu Grunde, so erweist sich die weitestgehende Anerkennung der Ausschlusswirkung gemeinschaftsrechtlicher Normen als wertungsmäßig konsistent. Grenzen unter dem Gesichtspunkt des Vertrauensschutzes bzw. der Rechtssicherheit könnten sich nur in Extremfällen ergeben, insbesondere in Fragen der strafrechtlichen Verantwortlichkeit ${ }^{56}$ oder wenn das nationale Recht keinerlei Verhaltensalternativen offen lässt. ${ }^{57} \mathrm{Im}$ Wortlaut der Rechtsprechung wird die maßgebliche Begründung bislang aber nicht hinreichend offen gelegt.

Für die Zukunft ist zu überlegen, ob nicht - entsprechend den dargestellten Anklängen in der Rechtsprechung des EuGH stärker nach den Auswirkungen auf die Verteilung der Regelungskompetenzen und auf den Vertrauensschutz bzw. die Rechtssicherheit gefragt werden sollte. Eine fast uneingeschränkte Orientierung an Vorrang und praktischer Wirksamkeit des Gemeinschaftsrechts erscheint nicht unzweifelhaft. Ggf. müssten daher die Grenzen der Ausschlusswirkung neu gefasst werden. $\mathrm{Zu}$ prüfen wäre insbesondere, ob der Wegfall von Rechten Einzelner, die an sich nach nationalem Recht begründet wären, unter dem Gesichtspunkt des Vertrauensschutzes bzw. der Rechtssicherheit der gemeinschaftsrechtlichen Begründung von Pflichten Einzelner gleichzustellen ist. Bejahendenfalls wäre weiter zu fragen, ob sich die Ausschlusswirkung dennoch mit den weniger gravierenden Auswirkungen auf die Verteilung der Regelungskompetenzen begründen ließe. Dem kann vorliegend nicht weiter nachgegangen werden.

\section{Zusammenfassung}

Im Zusammenhang mit dem Prinzip der unmittelbaren Wirkung steht die Ausschlusswirkung gemeinschaftsrechtlicher Vorschriften, die zur Unanwendbarkeit entgegenstehender nationaler Rechtsvorschriften führt. Der Verfasser sieht das vorherrschende Kriterium zur Bestimmung der Grenzen der Ausschlusswirkung - ob Pflichten Einzelner begründet werden als nicht ausreichend an. Vielmehr befürwortet er eine Analyse im Hinblick auf die Verteilung der Regelungskompetenzen und den Vertrauensschutz bzw. die Rechtssicherheit. Der EuGH scheint diesen Gesichtspunkten aber allenfalls in Ausnahmefällen Bedeutung zuzumessen. Im Regelfall zieht er der Ausschlusswirkung nur Grenzen, wenn andernfalls das zweistufige Regelungsverfahren der Richtlinie außer Kraft gesetzt würde.

\section{Summary}

Linked to the principle of direct effect is the blocking effect of community law that leads to the inapplicability of contravening national law. The author considers the general criterion if duties for individuals are generated - as insufficient to determine the limits of the blocking effect. The analysis should rather concentrate on the distribution of competencies and the protection of legitimate expectations or legal certainty. The ECJ though seems to attach only very limited significance to these aspects. In general he admits limits to the blocking effect only in the case of directives if the necessity to transpose community law by national law would be undermined.

\footnotetext{
55 Vgl. etwa EuGH Slg. 1995, I-4321 Rz. 17 - Spano; EuGH Slg. 1998, I- 4799 Rz. 36 - Silhouette International Schmied; EuGH Slg. 2000, I-6007 Rz. 9, $15 \mathrm{f}$. - Centrosteel.

56 Vgl. oben Fn. 42.

57 Vgl. oben Fn. 41.
}

\title{
Der Beitrag des Europäischen Gesellschaftsrechts zum System des Gemeinschaftsprivatrechts
}

\author{
Prof. Dr. Peter Jung, Basel
}

Der folgende Beitrag benennt die allgemein privatrechtlichen Grundsätze, Regelungen und Rechtsinstitute des geltenden Europäischen Gesellschaftsrechts und beschäftigt sich mit ihrer möglicherweise systembildenden Funktion für das übrige Gemeinschaftsprivatrecht. Dabei wird ersichtlich, daß dem Gesellschaftsrecht als dem Pionier und einem nach wie vor zentralen Gebiet des sekundären Gemeinschaftsprivatrechts zwar eine weitreichende Bedeutung für die Systembildung im Gemeinschaftsprivatrecht zukommt, das insoweit vorhandene Potential jedoch aus verschiedenen Gründen nicht ausgeschöpft wird.

\section{A. Einführung}

Grenzüberschreitende Tätigkeiten werden im Binnenmarkt vornehmlich von Gesellschaften entfaltet. So kann es nicht ver- wundern, daß sich die europäische Rechtsetzung im Bereich des Privatrechts zunächst dem Gesellschaftsrecht zugewandt hat ${ }^{1}$ und dieses bis heute einen wichtigen Platz im sekundären Privatrecht der Gemeinschaft einnimmt. ${ }^{2}$ Mit dem sog. NizzaKompromi $\beta$ in der Struktur- und Mitbestimmungsfrage bei der Europäischen Aktiengesellschaft (SE) ${ }^{3}$ konnte zu Beginn dieses Jahrzehnts zudem das größte Hindernis auf dem Weg zu weiteren Harmonisierungs- und Rechtsvereinheitlichungsmaßnah-

Siehe dazu Hallstein, RabelsZ 28 (1964), 221, 222 ff.

2 Siehe dazu etwa auch Grundmann, in Grundmann (Hrsg.), Systembildung und Systemlücken in Kerngebieten des Europäischen Privatrechts, 2000, S. 1, 26 ff. und Habersack, Europäisches Gesellschaftsrecht, 2. Aufl. 2003, Rn. 1.

3 Siehe dazu Art. 38 ff. der Vo EG/2157/2001 des Rates v. 8.10.2001 über das Statut der Europäischen Gesellschaft (SE), ABl. L 294/1 v. 10.11.2001 
men vermeintlich aus dem Weg geräumt werden. Seither ist der vornehmlich in den 1990er Jahren beklagte Stillstand im klassischen Bereich des Europäischen Gesellschaftsrechts ${ }^{3}$ einem verstärkten Aktionismus gewichen, der durch zahlreiche Anhörungen, ${ }^{4}$ die Bildung von Kommissionen, ${ }^{5}$ Aktionspläne ${ }^{6}$ und Vorschläge zu Rechtsakten ${ }^{7}$ gekennzeichnet ist.

Der Stand und die Perspektiven des Europäischen Gesellschaftsrechts ${ }^{8}$ sollen jedoch ebensowenig Gegenstand dieses Beitrags sein wie das innere System dieses Rechtsgebiets. ${ }^{9}$ Es sollen vielmehr die allgemein privatrechtlichen Elemente der gesellschaftsrechtlichen Richtlinien, Verordnungen und EuGHEntscheidungen herausgearbeitet und auf ihre systembildende Funktion für das übrige Gemeinschaftsprivatrecht hin untersucht werden. Hierzu werden die Beiträge des Europäischen Gesellschaftsrechts zu den allgemeinen Prinzipien, Instituten und Begriffen des Gemeinschaftsprivatrechts sowie zur Lösung allgemeiner Fragen der Gemeinschaftsrechtsetzung in den Blick genommen. Die mögliche Eignung einzelner Rechtsregeln oder Entscheidungen zur Systembildung wird dabei bewußt eher großzügig beurteilt, um auch auf aus heutiger Perspektive noch rudimentäre Konzepte zumindest hinweisen zu können. Denn das System des pointillistischen Gemeinschaftsprivatrechts ist mehr als jedes andere Privatrechtssystem im Werden begriffen und folglich unvollständig sowie fortwährenden Veränderungen unterworfen. Außerdem wird auch auf solche allgemein privatrechtlichen Elemente des Europäischen Gesellschaftsrechts eingegangen, die für dieses Rechtsgebiet nur am Rande eine Rolle spielen oder im Gesellschaftsrecht stillschweigend vorausgesetzt bzw. ohne eigenen Beitrag lediglich aufgenommen werden. Naturgemäß kann im Rahmen eines Aufsatzes auf viele Gesichtspunkte nur hingewiesen werden.

\section{B. Einzelne Systembeiträge des Europäischen Gesellschaftsrechts}

\section{Allgemein privatrechtliche Prinzipien im Europäischen Gesellschaftsrecht}

\section{Grundsatz der Privatautonomie}

Das bereits durch die Grundfreiheiten primärrechtlich vorgegebene ${ }^{10}$ Prinzip der Privatautonomie, d.h. der Grundsatz der Selbstgestaltung der Rechtsverhältnisse durch den einzelnen nach seinem Willen, ${ }^{11}$ prägt auch das Europäische Gesellschaftsrecht. Mit Art. 4 Abs. 2 der SE-RL ${ }^{12}$ hat der Grundsatz der Privatautonomie auch eine ausdrückliche Erwähnung gefunden. Bedeutsam ist er nicht nur für die meisten der hierfür disponierten, weil personengesellschaftsrechtlichen Regelungen der EWIV-VO, ${ }^{13}$ sondern auch etwa für die durchaus nicht selbstverständliche ${ }^{14}$ Abdingbarkeit der Handelndenhaftung nach Art. 7 Publizitäts-RL, ${ }^{15}$ Art. 16 Abs. 2 SE-VO und Art. 18 Abs. 2 SCE-VO. ${ }^{16}$ Es ist allerdings auch festzustellen, daß zwingende Regelungen, bedingt durch die gemeinschaftsrechtlichen

und die RL 2001/86/EG v. 8.10.2001 zur Ergänzung des SE-Statuts hinsichtlich der Beteiligung der Arbeitnehmer, ABl. L 294/22 v. 10.11.2001; dazu nur G. C. Schwarz, ZIP 2001, 1847, 1854 f. und Lutter, BB 2002, 1, $2 \mathrm{f}$. und $5 \mathrm{f}$.

4 Dazu nur Behrens, FS Mestmäcker 1996, S. 831 ff. und van Hulle, EWS 2000, 521, 522 f.; relativierend unter Einbeziehung der gerade in den 90er Jahren erfolgten kapitalmarktrechtlichen Veränderungen Grundmann, Europäisches Gesellschaftsrecht, 2004, Rn. 1141.
5 Siehe z.B. Europäische Kommission, Konferenz zu Binnenmarkt und Gesellschaftsrecht am 15. und 16.12.1997, Amt für amtliche Veröffentlichungen der Europäischen Gemeinschaften, 1998 sowie zum Konsultationspapier „Moderne gesellschaftsrechtliche Rahmenbedingungen in Europa“ der High Level Group of Experts on Corporate Law die Stellungnahme der Group of German Experts on Corporate Law, ZIP 2002, 1310 ff.; zur öffentlichen Konsultation betreffend Fragen der Corporate Governance http://europa.eu.int/comm/internal_market/de/company/ company/modern/index.htm und zur öffentlichen Konsultation betreffend Fragen der Sitzverlegung http://europa.eu.int/yourvoice/results/ transfer/index_de.htm.

6 Beispiele sind die SLIM-Arbeitsgruppe (dazu Europäische Kommission, Mitteilung an den Rat und das Europäische Parlament v. 4.2.2000 $\mathrm{KOM}(2000) 56$ endg. und v. 28.2.2000 KOM(2000) 104 endg. sowie Drygala, AG 2001, 291 ff.) und die High Level Group of Experts on Corporate Law (siehe dazu den Bericht v. 4.11.2002 http://www.euro pa.eu.int/comm/internal_market/en/company/company/modern/con sult/report_de.pdf).

7 Siehe Europäische Kommission, Mitteilung „Finanzdienstleistungen: Umsetzung des Finanzmarktrahmens: Aktionsplan“ v. 11.5.1999, KOM (1999) 232; Europäische Kommission, Mitteilung an den Rat und das Europäische Parlament „Rechnungslegungsstrategie der EU: Künftiges Vorgehen“ v. 13.6.2000, KOM(2000) 359; Europäische Kommission, Mitteilung an den Rat und das Europäische Parlament „Modernisierung des Gesellschaftsrechts und Verbesserung der Corporate Governance in der Europäischen Union - Aktionsplan“ v. 21.5.2003 KOM(2003) 284 endg.; Europäische Kommission, Mitteilung an den Rat und das Europäische Parlament zur Stärkung der Abschlußprüfung v. 21.5.2003, KOM (2003) 286 endg., abzurufen unter Markt/2003/10826.

8 Kommissionsvorschlag v. 18.11.2003 für eine Richtlinie über die Verschmelzung von Kapitalgesellschaften aus verschiedenen Mitgliedstaaten, $\operatorname{KOM(2003)~} 703$ (dazu Schulte-Hillen/Hirschmann, GPR 2003/04, $89 \mathrm{ff}$. und H. F. Müller, ZIP 2004, 1790 ff.); Kommissionsvorschlag v. 17.10.2003 für eine Richtlinie des Rates zur Änderung der RL 90/434/EWG des Rates v. 23.7.1990 über das gemeinsame Steuersystem für Fusionen, Spaltungen, die Einbringung von Unternehmensteilen und den Austausch von Anteilen, die Gesellschaften verschiedener Mitgliedstaaten betreffen, $\mathrm{KOM}(2003) 613$ endg.

9 Dazu u. a. Blaurock, ZEuP 1998, 460 ff.; Lutter, ZGR 2000, 1 ff.; van Hulle, EWS 2001, 521 ff.; Bayer, BB 2004, 1 ff.; Habersack, Europäisches Gesellschaftsrecht im Wandel, NZG 2004, 1 ff. und Drygala, ZEuP 2004, $337 \mathrm{ff}$.

10 Dazu u. a. Lutter, in Grundmann (Hrsg.), Systembildung und Systemlücken in Kerngebieten des Europäischen Privatrechts, 2000, S. 121 ff.; ders., ZGR 2000, 1 ff.; Grundmann, Europäisches Gesellschaftsrecht, 2004, Rn. 1135 ff.; Schön, RabelsZ 2000, 1 ff.; Merkt, RIW 2004, 1 ff.

11 Dazu nur Müller-Graff, Unternehmensinvestitionen und Investitionssteuerung im Marktrecht, 1984, S. 283 ff. und ders., Privatrecht und Europäisches Gemeinschaftsrecht - Gemeinschaftsprivatrecht, 2. Aufl. 1991, S. 17.

12 So Flume, Rechtsgeschäft, § 1, 1.

13 RL 2001/86/EG v. 8.10.2001 zur Ergänzung des SE-Statuts hinsichtlich der Beteiligung der Arbeitnehmer, ABl. L 294/22 v. 10.11.2001.

14 VO EWG/2137/85 des Rates v. 25.7.1985 über die Schaffung einer Europäischen wirtschaftlichen Interessenvereinigung (EWIV), ABl. L 199/1 v. 31.7.1985

15 Siehe dazu etwa die deutschen Leitentscheidungen RG v. 1.2.1910 RGZ 72, 401, 403 f. und BGH v. 14.3.1973 NJW 1973, 798.

16 RL 68/51/EWG des Rates v. 9.3.1968, ABl. L 65/8 v. 14.3.1968, zuletzt geändert durch RL 2003/58/EG v. 15.7.2003, ABl. L 221/13 v. 4.9.2003.

17 VO EG/1435/2003 des Rates v. 22.7.2003 über das Statut der Europäischen Genossenschaft (SCE), ABl. L 207/1 v. 18.8.2003 (dazu nur Mock, GPR 2003/04, 213 ff.). 
Regelungsschwerpunkte (Aktienrecht, Publizität, Vertretung, Rechnungslegung, Strukturmaßnahmen), im Europäischen Gesellschaftsrecht verhältnismäßig weit verbreitet sind. Bisweilen wird dieser Anteil zwingender Normen bei subsidiären Verweisen auf nationales Recht auch durch das jeweils maßgebliche Gesellschaftsstatut mit bestimmt. ${ }^{17}$

Eine prominente Einschränkung der Vertragsabschlußfreiheit findet sich im Übernahmerecht. Hier sollen unter bestimmten Voraussetzungen im Anschluß an eine Übernahme einerseits der Übernehmer von den verbliebenen Minderheitsaktionären den Verkauf ihrer Wertpapiere i.S.d. Art. 2 Abs. 1 lit. e Übernahme-RL ${ }^{18} \mathrm{zu}$ einem angemessenen Preis an sich (Ausschlußrecht nach Art. 15 Abs. 2 Übernahme-RL) und andererseits die Minderheitsaktionäre vom Übernehmer den Erwerb ihrer Wertpapiere zu einem angemessenen Preis verlangen können (Andienungsrecht nach Art. 16 Abs. 2 Übernahme-RL). Eine vergleichbare Anerkennung hat der Kontrahierungszwang auch beim allerdings lediglich als mitgliedstaatliche Option vorgesehenen Rückkauf von Wertpapieren mit Sonderrechten nach Art. 15 Fusions-RL ${ }^{19}$ und Art. 13 Spaltungs-RL ${ }^{20}$ erfahren. Einige Richtlinienbestimmungen können schließlich noch als Beispiele für eine sogar auf die vertragliche Äquivalenz bezogene Inhaltskontrolle gelten. ${ }^{21}$

Noch nicht abschließend geklärt sind im übrigen die Konsequenzen, die sich aus der weitreichenden Zulassung der institutionellen Haftungsbeschränkung auch im Europäischen Gesellschaftsrecht ${ }^{22}$ für die Zulässigkeit vertraglicher Haftungsbeschränkungen ergeben. Möglicherweise muß hier auch im Gemeinschaftsprivatrecht wegen des vorrangigen Grundsatzes der Gleichbehandlung von institutioneller und vertraglicher Haftungsbeschränkung dievertraglicheHaftungsbeschränkung entgegen Art. 3 Abs. 3 i.V.m. Anhang Nr. 1 lit. a Klausel-RL in stärkerem Maße der privatautonomen Gestaltung geöffnet werden. ${ }^{23}$

In ihren Voraussetzungen wird die Privatautonomie im Europäischen Gesellschaftsrecht insbesondere durch den sogleich noch näher zu behandelnden Informationsansatz gesichert. Daneben findet sich aber auch das von den verbraucherschutzrechtlichen Widerrufsrechten her bekannte Prinzip der Gewährung einer hinreichenden Bedenkzeit. So müssen die Inhaber von Wertpapieren einer Zielgesellschaft nach Art. 3 Abs. 1 lit. b Übernahme-RL im Interesse des Anlegerschutzes neben ausreichenden Informationen auch über genügend Zeit verfügen, um fundiert über das öffentliche Pflichtangebot bzw. freiwillige Angebot zur Übernahme eines Teils oder aller Wertpapiere entscheiden zu können.

\section{Informationsansatz und Offenlegungsgrundsatz}

Im Zentrum des Europäischen Gesellschaftsrechts stehen Regelungen zur Unternehmenspublizität. ${ }^{24}$ Die betroffenen Gesellschaften und etwaige Zweigniederlassungen sollen für ihre (potentiellen) Gesellschafter und Geschäftspartner rechtlich und wirtschaftlich durchschaubarer gemacht werden, indem sie umfangreichen und von den Mitgliedstaaten effektiv zu sanktionierenden ${ }^{25}$ Registrierungs- und Bekanntmachungspflichten unterliegen. Diese Pflichten beziehen sich als Register- ${ }^{26} \mathrm{Ge}-$ schäftsbrief- ${ }^{27}$ oder Hauspublizität ${ }^{28}$ auf die Rechtsform, ${ }^{29}$ den Satzungsinhalt, ${ }^{30}$ die Organmitgliedschaft und Vertretung, ${ }^{31}$ die Sachgründung, ${ }^{32}$ die Rechnungslegung ${ }^{33}$ sowie auf Umstrukturierungen $^{34}$ und Kontrolländerungen. ${ }^{35}$

Aufgrund ihres Inhalts sowie ihrer Rechtsnatur und Intensität können die gesellschaftsrechtlichen Publizitätspflichten jedoch nur zum Teil auf Einzelunternehmer und gar nicht auf
18 So unterliegt etwa eine SE mit Verwaltungssitz in Deutschland einer erheblichen Satzungsstrenge nach Art. 9 Abs. 1 lit. b SE-VO und lit. c iii) SE-VO i.V.m. § 23 Abs. 5 AktG; entsprechendes gilt nach Art. 8 Abs. 1 lit. b und lit. c iii) SCE-VO i.V.m. § 18 Abs. 2 GenG für die SCE.

19 RL 2004/25/EG des Europäischen Parlaments und des Rates v. 21.4.2004 betreffend Übernahmeangebote, ABl. L 142/12 v. 30.4.2004.

20 RL 78/855/EWG v. 9.10.1978 betreffend die Verschmelzung von Aktiengesellschaften, ABl. L 295/36 v. 20.10.1978.

21 RL 82/891/EWG v. 17.12.1982 betreffend die Spaltung von Aktiengesellschaften ABl. L 378/47 v. 31.12.1982.

22 Siehe Art. 5 Abs. 2 S. 2 und 3 Spaltungs-RL („In diesem Fall haben sie Anspruch auf ein dem Wert ihrer Aktien entsprechendes Entgelt. Sofern hierüber keine Einigung erzielt wird, muß das Entgelt durch ein Gericht festgelegt werden können.“), Art. 5 Abs. 4 Übernahme-RL („Als angemessener Preis gilt der höchste Preis, der ...") und Art. 15 Abs. 5 S. 1 Übernahme-RL („Die Mitgliedstaaten stellen sicher, daß eine angemessene Abfindung garantiert wird.“).

23 Siehe zu der nach Art. 2 Abs. 1 Einpersonen-GmbH-RL nunmehr auch für die Einpersonen-GmbH und damit erst Recht für die anderen Kapitalgesellschaften geltenden Haftungsbeschränkung den Erwägungsgrund 5 der Einpersonen-GmbH-RL: „Einzelunternehmern in der gesamten Gemeinschaft sollte das rechtliche Instrument einer Gesellschaft mit Haftungsbeschränkung geboten werden“; dazu auch Schön, RabelsZ 2000, 1, 14.

24 Dazu A. Bruns, Haftungsbeschränkung und Mindesthaftung, 2003, S. $142 \mathrm{f}$.

25 Siehe zum Informationsansatz des Europäischen Gesellschaftsrechts etwa Europäische Kommission, Mitteilung an den Rat und das Europäische Parlament „Modernisierung des Gesellschaftsrechts und Verbesserung der Corporate Governance in der Europäischen Union - Aktionsplan“ v. 21.5.2003 KOM(2003) 284 endg., S. 15 f. und 26 f. sowie den Bericht der Hochrangigen Expertengruppe v. 4.11 .2002 (Fn. 6), S. 34 f.; dazu auch Schön, RabelsZ 2000, 1, 25 ff. und Grundmann, Europäisches Gesellschaftsrecht, 2004, Rn. 1140.

26 Zur Sanktionierungspflicht nach Art. 6 Publizitäts-RL EuGH v. 4.12.1997 Rs. C-97/96, Slg.1997, I-6843 (Daihatsu); dazu auch Leible, ZHR 162 (1998), $594 \mathrm{ff}$.

27 Siehe dazu etwa Art. 2 f. Publizitäts-RL; Art. 6 ff. EWIV-VO.

28 Siehe dazu etwa Art. 4 Publizitäts-RL, Art. 6 Zweigniederlassungs-RL und Art. 25 EWIV-VO.

29 Siehe dazu etwa Art. 47 Abs. 1 UAbs. 2 Bilanz-RL und Art.11 FusionsRL.

$30 \mathrm{Zu}$ den nach Europäischem Gesellschaftsrecht verbindlichen Rechtsformzusätzen in der Gesellschaftsfirma siehe Art. 1 Abs. 1 S. 2 KapitalRL; Art. 5 lit. a EWIV-V0; Art. 11 Abs. 1 SE-VO sowie Art. 1 Abs. 2 S. 3 und 10 Abs. 2 SCE-VO.

31 Siehe dazu etwa Art. 2 Abs. 1 lit. a Publizitäts-RL sowie Art. 2 und 3 Kapital-RL.

32 Siehe dazu etwa Art. 2 Abs. 1 lit. d.

33 Siehe dazu etwa Art.11 Kapital-RL.

34 Siehe dazu etwa Art. 47 ff. Bilanz-RL und 38 f. Konzernbilanz-RL; auch das im Europäischen Rechnungslegungsrecht vorrangige true and fair view-Prinzip erklärt sich aus dem Bedürfnis des Anlegers nach ordnungsgemäßer Information über seine Chancen und Risiken (dazu Grundmann, Europäisches Gesellschaftsrecht, 2004, Rn. 48).

35 Siehe dazu etwa Art. 6 und 9 Fusions-RL, Art. 7 Spaltungs-RL sowie Art. 8 Abs. 2-6 SE-V0; näher zum Informationsansatz in der Fusions-RL K. Riesenhuber, NZG 2004, 15, $19 \mathrm{ff}$.

36 Siehe dazu etwa Art. 3 Abs. 1 lit. b und Art. 6 Abs. 2 Übernahme-RL. 
Privatpersonen übertragen werden. Informationsasymmetrien sollen im Gesellschaftsrecht anders als im übrigen Privatrecht vorrangig kollektiv durch Publizität und nicht durch eine individualvertragliche Aufklärung beseitigt werden. ${ }^{37}$ Ein am Markt tätiger Unternehmer und insbesondere eine Kapitalgesellschaft können sich zudem weniger auf Geheimhaltungsinteressen berufen als eine Privatperson. ${ }^{38}$ Im Unternehmensrecht wird die Verletzung von Informationspflichten schließlich eher durch eine Haftung für unterlassene und fehlerhafte Information $^{39}$ und weniger als sonst im Vertragsrecht durch eine Bindung an die gegebene Information ${ }^{40}$ sanktioniert. ${ }^{41}$

Selbst als einer spezifisch unternehmensrechtlichen Regelung kommt den Bestimmungen zur Publizität jedoch für das allgemeine Gemeinschaftsprivatrecht insofern eine Bedeutung $\mathrm{zu}$, als sie eine besonders intensive Ausprägung des gemeinschaftsrechtlichen Informationsansatzes darstellen. Danach soll die Informationsgewährung nicht nur die Voraussetzungen für eine interessengerechte privatautonome Rechtsgestaltung schaffen ${ }^{42}$ sondern auch den Rechtsverkehr zwischen Angehörigen verschiedener Mitgliedstaaten befördern. ${ }^{43}$ Daher sind auch Angaben zu machen, die sich ohne weiteres aus den nationalen Rechtsvorschriften ergeben oder offenkundig erscheinen, da von Außenstehenden keine vollständige Kenntnis der Rechtsvorschriften eines anderen Mitgliedstaates oder der dort herrschenden Handelsbräuche erwartet werden kann. ${ }^{44}$ Gerade auch im Zusammenhang mit der Rechtfertigung nationaler Beschränkungen der gesellschaftsrechtlichen Niederlassungsfreiheit hat der EuGH verdeutlicht, daß die Auferlegung von Informationspflichten als milderes Mittel im Vergleich zu zwingenden Schutzregelungen in Betracht gezogen werden muß. ${ }^{45}$

\section{Verkehrsschutz}

Der Vertrauensschutz nimmt im Gesellschaftsrecht als einem Teil des Unternehmensrechts eine besondere Stellung ein. ${ }^{46}$ Hierzu tragen zunächst generell vertrauensschützende Rechtsinstitute bei. $\mathrm{Zu}$ diesen gehört insbesondere der auch außerhalb des Unternehmensgegenstands Geltung beanspruchende Grundsatz der nach außen hin unbeschränkten und unbeschränkbaren Vertretungsmacht von Mitgliedern des Vertretungsorgans. ${ }^{47}$ Einen wichtigen Beitrag zum allgemeinen Verkehrsschutz leistet aber auch die Zurückdrängung der Nichtigkeit durch die Reduzierung von Nichtigkeitsgründen, ${ }^{48}$ die Schaffung von Heilungsmöglichkeiten ${ }^{49}$ und die Begrenzung von Nichtigkeitswirkungen. ${ }^{50}$ Darüber hinaus bestehen noch Sonderregelungen zur materiellen Registerpublizität, die dem Dritten den Schutz nur bei Kenntnis versagen. ${ }^{51}$ Die Rechtsfolgen eines der Handelsregistereintragung widersprechenden Rechtsscheins richten sich hingegen immer noch nach nationalem Recht. ${ }^{52}$

Da der weitreichende unternehmensrechtliche Vertrauensschutz nur mit dem besonderen Bedürfnis des Handelsverkehrs nach Transaktionssicherheit und im Bereich der materiellen Registerpublizität mit der Herstellung eines materiellrechtlichen Registerzwangs gerechtfertigt werden kann, ${ }^{53}$ kommt eine Ausdehnung der genannten Regelungen auf das allgemeine Zivilrecht allerdings nur sehr eingeschränkt in Betracht. BeiBeschränkungen der Vertretungsmacht ist lediglich in Fällen, in denen ein den unternehmensrechtlichen SituationenvergleichbarerbesondererVertrauenstatbestand geschaffen wurde, ${ }^{54}$ ein entsprechender Verkehrsschutz vorstellbar. Demgegenüber könnte die $\mathrm{Zu}-$ rückdrängung der Unwirksamkeit von Rechtsgeschäften durchaus Modellcharakter für andere Dauerschuldverhältnisse besitzen.

\section{Grundsatz von Treu und Glauben}

\section{a) Mitgliedschaftliche Treuepflichten}

Aus der Mitgliedschaft in der Gesellschaft ergeben sich zunächst für jeden Gesellschafter unabhängig von der Gesellschaftsform eine Reihe von Verhaltenspflichten, die unter dem Begriff der Treuepflicht zusammengefaßt werden. ${ }^{55}$ Sieht man einmal vom sogleich noch gesondert zu behandelnden Fall der mißbräuchlichen Gesellschafterklage ab, spielt diese Form der Treuepflicht im Europäischen Gesellschaftsrecht bislang jedoch kaum eine Rolle, da sie die für das Gemeinschaftsrecht weniger wichtigen Innenbeziehungen der Gesellschafter untereinander und zur Gesellschaft betrifft. Ein Anwendungsfall des Prinzips könnte aber etwa in der Regelung des Art. 6 Abs. 3 lit. i Über-

37 Dazu auch Grundmann, Europäisches Gesellschaftsrecht, 2004, Rn. 229.

38 Dazu $v$. Caemmerer, in: Frankfurter Publizitätsgespräch, S. 141 ff.; Wimmer, Bilanzpublizität, S. 29 ff.; Windbichler, CR 1988, 447, 449; Hirte, CR 1990, 631, 633.

39 Siehe dazu etwa Art. 5 f. Publizitäts-RL; Art. 20 f. Fusions-RL und Art. 26 Prüferbefähigungs-RL.

40 Siehe aber auch Art. 3 Abs. 6 UAbs. 2, Art. 3a Abs. 4 UAbs. 2 S. 2 und Art. 8 Publizitäts-RL, die Regelungen zur positiven Publizität und damit zur Bindung an die gegebene Information enthalten.

41 Dazu auch Grundmann, Europäisches Gesellschaftsrecht, 2004, Rn. 229.

42 Dazu nur M. Wolf, Rechtsgeschäftliche Entscheidungsfreiheit, S. $101 \mathrm{f}$.

43 EuGH v. 12.11.1974, Rs. C-32/74, Slg. 1974, 1201, Rz. 6 (Haaga).

44 So EuGH v. 12.11.1974, Rs. C-32/74, Slg.1974, 1201, Rz. 6 (Haaga), wonach die von Art. 2 Abs. 1 lit. d S. 2 Publizitäts-RL geforderten Angaben zur Vertretungsbefugnis auch dann zu machen sind, wenn nur ein einziger vertretungsberechtigter Organwalter bestellt ist und somit ausschließlich eine Einzelvertretungsbefugnis in Betracht kommt.

45 EuGH v. 9.3.1999, Rs. C-212/97, Slg. 1999, I-1459 Rz. 36 (Centros).

46 Grundlegend für das deutsche Recht Canaris, Die Vertrauenshaftung im deutschen Privatrecht, 1971, S. 150 ff.

47 Zur grundsätzlichen Vertretungsmacht von Gesellschaftsorganen bei Rechtshandlungen auch außerhalb des Unternehmensgegenstands Art. 9 Publizitäts-RL, Art. 20 Abs. 1 EWIV-VO und Art. 47 Abs. 2 SCE-V0; mögliche Ausnahmen sind das Handeln außerhalb der gesetzlichen Zuständigkeitsordnung, die Kenntnis und das (wohl nur bei Evidenz des Mißbrauchs gegebene) Kennenmüssen (dazu näher Habersack, Europäisches Gesellschaftsrecht, 2. Aufl. 2003, Rn. 102 ff.).

48 Siehe dazu Art.11 Publizitäts-RL, Art.22 Fusions-RL und Art.19 Spaltungs-RL.

49 Siehe dazu Art. 15 Abs. 1 S. 2 und Art. 32 Abs. 1 EWIV-VO.

50 Siehe dazu Art. 12 Publizitäts-RL.

51 Art. 3 Abs. 5 bis 7 Publizitäts-RL (negative und positive Registerpublizität); Art. 3a Abs. 4 Publizitäts-RL (Ausdehnung des Vertrauensschutzes auf fakultativ offen gelegte Übersetzungen); Art. 8 Publizitäts-RL (bei ordnungsgemäßer Offenlegung können Mängel der Organbestellung Dritten nur bei deren Kenntnis entgegengesetzt werden); Art. 9 Abs. 1 und Art. 15 Abs. 3 EWIV-VO (Verweis auf Art. 3 Abs. 5 und 7 der Publizitäts-RL); Art.14 Abs. 3 EWIV-VO (Sonderregelung zum Vertrauensschutz bei Sitzverlegung).

52 Siehe dazu EuGH v. 20.9.1988, Rs.C-136/87, Slg. 1988, 4665, 4682 Rz. 13 ff. (Ubbink Isolatie BV/Dak -en Wandtechniek BV).

53 Dazu nur Merkt, Unternehmenspublizität - Offenlegung von Unternehmensdaten als Korrelat der Marktteilnahme, 2001, S. 231 ff. und 334.

54 Beispiele bilden die den §§ 171 ff., 405, 409 BGB zugrundeliegenden Fallgestaltungen.

55 Siehe dazu nur für das deutsche Recht BGH v. 5.6.1975 BGHZ 65, 15, 18 f. und Lutter, AcP 180 (1980), 84, 97 ff. und 102 ff. 
nahme-RL gesehen werden, wonach die Unterlage $\mathrm{zu}$ einem öffentlichen Übernahmeangebot auch die Absichten des Bieters in Bezug auf die künftige Geschäftstätigkeit zumindest der Zielgesellschaft enthalten muß. Denn diese weitreichende Offenlegungspflicht läßt sich letztlich nur mit einer gesteigerten Treuepflicht des die Gesellschaft künftig kontrollierenden Bieters rechtfertigen. ${ }^{56}$ Sofern die mitgliedschaftliche Treuepflicht nicht ausnahmsweise auf den privaten Bereich der Gesellschafter ausstrahlt, ${ }^{57}$ hat sie jedoch keine außergesellschaftsrechtliche Bedeutung.

\section{b) Organschaftliche Treuepflichten}

Die organschaftliche Treuepflicht, nach der die Organmitglieder aufgrund ihrer gesteigerten Verfügungsmacht über fremdes Vermögen $^{58}$ die Interessen der Gesellschaft bestmöglich zu fördern und eine Schädigung derselben zu unterlassen bzw. zu verhindern haben, ${ }^{59}$ hat ihren gemeinschaftsrechtlichen Ausdruck etwa in der Bindung an das Interesse der gesamten Gesellschaft nach Art. 3 Abs. 1 lit. c Übernahme-RL und in der Verschwiegenheitspflicht nach Art. 49 SE-VO bzw. Art. 49 SCEVO gefunden. Auch das im Zusammenhang mit der Regelung zum Selbstkontrahieren in Art. 5 Abs. 2 Einpersonen-GmbH$\mathrm{RL}^{60}$ angesprochene ${ }^{61}$ Prinzip des Drittvergleichs (,at arms length-Prinzip“) ist ein wichtiges Element organschaftlicher Treuebindung. ${ }^{62}$ Weitere einschlägige Regelungen finden sich im Kommissionsentwurf für eine Fünfte Richtlinie des Rates über die Struktur der Aktiengesellschaft (Struktur-RL), ${ }^{63}$ der hier nicht näher zu behandeln ist, da er immer noch auf Eis liegt und voraussichtlich durch die aktuellen Pläne zur Corporate Governance abgelöst wird.

Im Gegensatz zur spezifisch gesellschaftsrechtlichen mitgliedschaftlichen Treuepflicht läßt sich die organschaftliche Treuepflicht als Ausprägung eines generellen Prinzips der Treuebindung von Fremdvermögensverwaltern begreifen. ${ }^{64}$ Insoweit kann daher das verhältnismäßig weit entwickelte europäische Recht der Gesellschaftsorgane neben der Handelsvertreterrichtlinie auch als Modell für andere Formen der Wahrnehmung fremder Interessen (Vermittlungs-, Beratungs-, Betreuungs- und Treuhandverhältnisse) herangezogen werden. ${ }^{65}$ Dies gilt insbesondere für die Wahrung des at arms length-Prinzips.

\section{c) Verbot des Rechtsmißbrauchs}

Die einzelfallbezogene Einschränkung einer Rechtsausübung unter dem Gesichtspunkt des individuellen oder institutionellen Rechtsmißbrauchs ${ }^{66}$ kann als ein gemeineuropäischer Privatrechtsgrundsatz gelten. ${ }^{67}$ Auch im Europäischen Gesellschaftsrecht findet sich in Art. 11 der SE-RL ${ }^{68}$ eine dem Verbot des institutionellen Rechtsmißbrauchs zuzuordnende Regelung, wonach die Mitgliedstaaten im Einklang mit den gemeinschaftlichen Rechtsvorschriften dafür Sorge tragen sollen, daß eine SE nicht dazu mißbraucht wird, Arbeitnehmern Beteiligungsrechte zu entziehen oder vorzuenthalten. Einen wichtigen Beitrag zur Fortentwicklung des auch für das Gemeinschaftsrecht bedeutsamen Rechtsprinzips hat der EuGH zudem anhand gesellschaftsrechtlicherFällegeleistet. ${ }^{69}$ Zwarkennt das Gemeinschaftsrechtbereits seit längerem die Einschränkung von Grundfreiheiten unter dem Aspekt der Umgehung von mit dem Gemeinschaftsrecht vereinbaren nationalen Rechtsvorschriften ${ }^{70}$ und im Bereich des unmittelbar Rechtspositionen begründenden Verordnungsrechts. ${ }^{71}$ Die Anerkennung des Rechtsmißbrauchsverbots als eines allgemei- nen Grundsatzes des Gemeinschaftsrechts blieb jedoch ebenso fraglich $^{72}$ wie sein genauer Inhalt. ${ }^{73}$

56 Dazu näher Burgard, AG 1992, 41, 47 ff.; ders., Die Offenlegung von Beteiligungen, Abhängigkeits- und Konzernlagen bei der Aktiengesellschaft, 1990, S. 65 ff.; ferner Jung, Der Unternehmergesellschafter als personaler Kern der rechtsfähigen Gesellschaft, 2002, S. 245 f.

57 Siehe dazu Jung (Fn. 56), S. 202 f.

$58 \mathrm{Zu}$ dieser heute nicht nur im deutschen Recht vorherrschenden Rechtfertigung der organschaftlichen Treuepflicht etwa bereits Mestmäcker, Verwaltung, Konzerngewalt und Rechte der Aktionäre, 1958, S. 214.

59 Siehe dazu für das deutsche Recht etwa Lutter, ZHR 145 (1981), 224, 236 und Scheffler, DB 1994, 793, 795.

60 RL 89/667/EWG des Rates v. 21.12.1989 betreffend Gesellschaften mit beschränkter Haftung mit einem einzigen Gesellschafter, ABl. L 395/40 v. 30.12.1989.

61 Nach Art. 5 Abs. 2 Einpersonen-GmbH-RL können die Mitgliedstaaten Geschäfte, die i.S.v. Abs. 2 zu den laufenden Geschäften gehören und unter normalen Bedingungen abgeschlossen werden, vom Dokumentationserfordernis nach Abs. 1 befreien.

62 Siehe dazu für das deutsche Recht etwa Scholz/U.H.Schneider, § 43 Rn. 150 ff.; KK/Mertens, § 93 Rn. 62; Hopt, ZGR 2004, 1, 10 f.

63 Siehe dazu etwa Art. 10 Abs. 2 S. 2 (Stimmrechtsausschluß bei Interessen- oder Pflichtenkollision) und Art. 10a Abs. 2 (Bindung der Organmitglieder an die Interessen des Unternehmens, der Aktionäre und der Arbeitnehmer) der dritten Änderung des Vorschlags für eine Fünfte Richtlinie des Rates über die Struktur der Aktiengesellschaft sowie die Befugnisse und Verpflichtungen ihrer Organe v. 20.11.1991 KOM(91) 372 endg., ABl. C 321/9 v. 12.12.1991.

64 So auch im deutschen Recht etwa BGH v. 20.2.1995 129, 30, 34; KK/ Mertens, § 93 Rn. 6 und 57 ff.

65 Vgl. dazu jüngst im deutschen Recht Hopt, ZGR 2004, 1 ff.

66 Die in der deutschen Dogmatik verbreitete Unterscheidung zwischen individuellem und institutionellem Rechtsmißbrauch hat im Gemeinschaftsrecht bislang offenbar keinen Niederschlag gefunden.

67 Siehe etwa A. Zimmermann, Das Rechtsmißbrauchsverbot im Recht der Europäischen Gemeinschaften, 2002, S. 68 ff.; überblicksartig Fleischer, JZ 2003, 865 ff.; einschränkend GA Tesauro, Rs C-367/96, Slg. 1998, I-2843 Rz. 22 (Kefalas).

68 RL 2001/86/EG v. 8.10.2001 zur Ergänzung des SE-Statuts hinsichtlich der Beteiligung der Arbeitnehmer, ABl. L 294/22 v. 10.11.2001.

69 EuGH v. 12.3.1996 Rs.C-441/93, Slg.996, I-1347 (Pafitis); EuGH v. 12.5.1998, Rs C-367/96, Slg. 998, I-2843 (Kefalas) mit krit. Anm. Ranieri, ZEuP 2001, 165 ff.; EuGH v. 23.3.2000, Rs C-373/97, Slg. 2000, I-1705 (Diamantis); EuGH v. 9.3.1999, Rs. C-212/97, Slg.999, I-1459 (Centros); dazu näher Schön, FS Wiedemann 2002, S. 1271 ff.; Schmidt-Kessel, Jahrbuch Junger Zivilrechtswissenschaftler, Prinzipien des Privatrechts und Rechtsvereinheitlichung (2000), S. 61 ff. und Fleischer, JZ 2003, 855 ff.

70 St. Rspr. seit EuGH v. 3.12.1974, Rs. C-33/74, Slg. 974, 1299, Rz. 13 (Van Binsbergen); eingehend Ottersbach, Rechtsmißbrauch bei den Grundfreiheiten des Europäischen Binnenmarkts, 2001; siehe speziell für den Bereich des Gesellschaftsrechts EuGH v. 9.3.1999, Rs.C-212/97, Slg. 999, I-1459, Rz. 23 ff. m.w.N. (Centros) und EuGH v. 30.9.2003 Rs. C-167/01 (Inspire Art), NJW 2003, 3331 = AG 2003, 680, Rz. 136 ff., wonach die Ausübung der Niederlassungsfreiheit auch dann nicht rechtsmißbräuchlich bzw. betrügerisch ist, wenn damit gezielt einem als strenger angesehenen Recht aus dem Weg gegangen wird.

71 Siehe dazu etwa EuGH v. 21.6.1988, Slg. 988, 3161, Rz. 43 (Lair).

72 Vgl. dazu etwa noch ausdrücklich ablehnend GA Tesauro, Rs C-367/96, Slg. 998, I-2843 Rz. 18 ff. (Kefalas).

73 Siehe dazu etwa die lediglich pauschalen Hinweise in EuGH v. 3.12.1974, Rs. C-33/74, Slg. 974, 1299, Rz.13 (Van Binsbergen) und EuGH v. 21.6.1988, Slg. 988, 3161, Rz. 43 (Lair). 
Mit den gesellschaftsrechtlichen Entscheidungen Kefalas und Diamantis hat sich hingegen das Verbot der mißbräuchlichen Berufung auf Normen des Gemeinschaftsrechts als allgemeiner Grundsatz des Gemeinschaftsrechts und dies auch für den Bereich des Richtlinienrechts etabliert. ${ }^{74}$ Zwar billigte es der EuGH in den genannten Fällen den vorlegenden griechischen Gerichten zu, die Berufung der Kläger auf Art. 25 Abs. 1 der Kapital-RL ${ }^{75}$ unter den Vorbehalt des nationalen Rechtsmißbrauchsverbot von Art. $281 \mathrm{GrZGB}^{76}$ zu stellen, ${ }^{77}$ obwohl die einschlägige Richtlinienbestimmung wegen ihrer unzureichenden Umsetzung in griechisches Recht ausnahmsweise unmittelbar anwendbar war. ${ }^{78}$ Er machte jedoch zugleich deutlich, daß die Anwendung des nationalen Rechtsmißbrauchsverbots nicht dem Ziel der gemeinschaftsrechtlichen Regelung sowie dessen effektiver und einheitlicher Verwirklichung zuwiderlaufen dürfe. ${ }^{79}$ Ein Rechtsmißbrauch dürfe daher nur dann angenommen werden, wenn der sich auf Art. 25 Abs. 1 der Kapital-RL berufende Aktionär eine Klage erhöbe, um widerrechtliche und mit dem Zweck dieser Vorschrift offensichtlich unvereinbare Vorteile zu Lasten der Gesellschaft zu erlangen. ${ }^{80}$ Damit wird die formale Anwendung des Art. 281 GrZGB durch die vorlegenden Gerichte inhaltlich durch das allerdings noch lückenhafte gemeinschaftsrechtliche Rechtsmißbrauchsverbot bzw. die allgemeinen und fallbezogenen Auslegungsrichtlinien des EuGH dominiert und auf diese Weise der Vorrang des Gemeinschaftsrechts gewahrt. ${ }^{81}$ Dies ist auch deshalb geboten, weil nach der offensichtlich auch im Gemeinschaftsrecht dominierenden Innentheorie, ${ }^{82}$ wonach der Rechtsmißbrauch bereits eine innere Beschränkung des betreffenden Rechts darstellt, der EuGH die Reichweite der gemeinschaftsrechtlich begründeten Rechtsposition im Rahmen einer teleologischen Auslegung festzulegen hat.

Durch die den vorlegenden Gerichten gegebenen Auslegungshinweise hat der EuGH zugleich den Tatbestand des gemeinschaftsrechtlichen Rechtsmißbrauchs präzisiert. Danach genügt unter Verzicht auf ein subjektives Element mit dem zweckwidrigen Ausnutzen einer durch Gemeinschaftsrecht erlangten Rechtsposition ein rein objektiver Mißbrauch. ${ }^{83}$ Besonders hervorgehoben wird zudem die Bedeutung der effektiven und gleichmäßigen Anwendung des Gemeinschaftsrechts für den gemeinschaftsrechtlichen Mißbrauchstatbestand, so daß etwa eine Nichtigkeitsklage auch dann erhoben werden kann, wenn die Gesellschaft und damit auch der Kläger von der angegriffenen Kapitalerhöhung profitiert hat. ${ }^{84}$ Ebensowenig könne dem Kläger entgegengehalten werden, er habe das Bezugsrecht für die anläßlich derstreitigen Kapitalerhöhung ausgegebenen neuen Aktien nicht ausgeübt.

Zwar werden vom EuGH noch keine Fallgruppen des Rechtsmißbrauchs gebildet bzw. gar benannt, doch enthalten die Entscheidungen Aussagen zu verschiedenen, etwa auch in der deutschen Rechtsordnung anerkannten Fällen des Rechtsmißbrauchs. Wenn beispielsweise in der Entscheidung Kefalas dem vorlegenden Gericht angesichts hinreichender Anhaltspunkte die Prüfung gestattet wird, ob der sich auf Art. 25 Abs. 1 der Kapital-RL berufende Aktionär eine Klage auf Feststellung der Ungültigkeit der Kapitalerhöhung zu dem Zweck erhoben hat, widerrechtliche Vorteile zum Nachteil der Gesellschaft zu erlangen, ${ }^{85}$ so ist damit der Unterfall des rücksichtslos eigennützigen Verhaltens angesprochen. In der Entscheidung Diamantis wird ferner erörtert, ob ein Rechtsmißbrauch nicht allein deshalb angenommen werden kann, weil der auf Feststellung der Nichtigkeit der Kapitalerhöhung Klagende letztlich von der Kapitalerhöhung profitiert habe oder weil er sein Bezugsrecht nicht ausgeübt habe. Thematisiert wird auch, ob die Berufung auf die fehlende Zustimmung der Hauptversammlung zu einer Kapitalerhöhung nicht deshalb rechtsmißbräuchlich sein könne, weil der Nichtigkeitskläger zuvor einen Antrag auf Einleitung eines diese Zustimmung nach griechischem Recht gerade entbehrlich machenden Verfahrens gestellt habe. Insoweit wurde unausgesprochen der Tatbestand widersprüchlichen Verhaltens als ein möglicher Ansatzpunkt für rechtsmißbräuchliches Verhalten anerkannt, ${ }^{86}$ wenn auch das Verhalten des Klägers im konkreten Fall völlig zu Recht nicht als widersprüchlich und rechtsmißbräuchlich angesehen wurde. ${ }^{87}$ Ein Rechtsmißbrauch

74 EuGH v. 12.5.1998, Rs C-367/96, Slg. 998, I-2843, Rz. 20 (Kefalas) und EuGH v. 23.3.2000, Rs C-373/97, Slg. 000, I-1705, Rz. 33 (Diamantis): , ist die mißbräuchliche oder betrügerische Berufung auf Gemeinschaftsrecht nicht gestattet“; ausdrücklich für die Qualifikation als allgemeines Prinzip auch GA La Pergola, Rs. C-212/97, Slg. 999, I-1459, Rz. 20 (Centros); dazu auch Schmidt-Kessel, Jahrbuch Junger Zivilrechtswissenschaftler, Prinzipien des Privatrechts und Rechtsvereinheitlichung (2000), S. 61, 70 f. und 79 f. sowie Fleischer, JZ 2003, 865, 868 ff.; zurückhaltender A. Zimmermann, Rechtsmißbrauchsverbot, 2002, S. 202 ff.

75 Zweite Richtlinie 77/91/EWG des Rates vom 13.12.1976, ABl. L 26/1 v. 30.1.1977, zuletzt geändert durch Richtlinie 92/101/EWG des Rates vom 23.11.1992, ABl. L 347/64 v. 28.11.1992.

76 Nach Art. 281 GrZGB ist „die Ausübung eines Rechts ... unzulässig, wenn sie die sich aus Treu und Glauben, aus den guten Sitten oder aus dem sozialen oder wirtschaftlichen Zweck des betreffenden Rechts ergebenden Schranken offensichtlich überschreitet“.

77 EuGHv. 12.5.1998, Rs C-367/96, Slg. 998,I-2843, Rz. 21 (Kefalas);EuGHv. 23.3.2000, Rs C-373/97, Slg. 000, I-1705, Rz. 34 (Diamantis); hierzu krit. Ranieri, ZEuP 2001, 165 ff. und Fleischer, JZ 2003, 865, 873 f.; zur möglichen Heranziehung der nationalen Rechtsmißbrauchsverbote zum Schutz von Richtlinienbestimmungen zur Sachgründung gegen Umgehung auch bereits GA Tesauro, Rs. C-83/91, Slg. 992, I-4919 Rz. 21 (Meilicke).

78 Dazu bereits EuGH v. 30.5.1991, verb. Rs. C-19/90 und C-20/90, Slg. 991, I-2691, Rn. 28 (Karella und Karellas) sowie EuGH v. 24.3.1992, Rs. C-381/89, Slg. 992, I-2111, Rn. 35 (Syndesmos Melon tis Eleftheras Evangelikis Ekklisias u. a.).

79 EuGH v. 12.5.1998, Rs C-367/96, Slg. 998, I-2843, Rz. 22 (Kefalas); EuGH v. 23.3.2000, Rs C-373/97, Slg. 000, I-1705, Rz. 34 (Diamantis).

80 EuGH v. 12.5.1998, Rs C-367/96, Slg. 998, I-2843, Rz. 28 (Kefalas); EuGH v. 23.3.2000, Rs C-373/97, Slg. 000, I-1705, Rz. 33 (Diamantis).

81 Dazu bereits GA Tesauro, Rs. C-83/91, Slg. 992, I-4919 Rz. 21 (Meilicke); näher Schmidt-Kessel, Jahrbuch Junger Zivilrechtswissenschaftler, Prinzipien des Privatrechts und Rechtsvereinheitlichung (2000), S. 61, 75 ff.; ebenso Fleischer, JZ 2003, 865, 873 (mehr Schein als Sein).

82 Siehe dazu GA Tesauro, Rs C-367/96, Slg. 998, I-2843, Rz. 25 (Kefalas); GA La Pergola, Rs. C-212/97, Slg. 999, I-1459, Rz. 20 (Centros) und GA Saggio, Rs C-373/97, Slg. 000, I-1705, Rz. 25 (Diamantis); siehe aber auch zu der im Bereich der Grundfreiheiten vorherrschenden Außentheorie GA Lenz, Rs. C-23/93, Slg. 994, I-4795, Rz. 35 (TV 10); speziell zur dogmatischen Einordnung des Rechtsmißbrauchseinwands im Europäischen Gesellschaftsrecht Schön, FS Wiedemann, 2002, S. 1271, $1282 \mathrm{ff}$.

83 Inwieweit dies generell für das gemeinschaftsrechtliche Mißbrauchsverbot gilt, ist allerdings fraglich (dazu Fleischer, JZ 2003, 865, 872).

84 EuGH v. 12.5.1998, Rs C-367/96, Slg. 998, I-2843, Rz. 23 ff. (Kefalas).

85 EuGH v. 12.5.1998, Rs C-367/96, Slg. 1998, I-2843, Rz. 28 (Kefalas).

86 Vgl. dazu auch GA Saggio, Rs C-373/97, Slg. 2000, I-1705, Rz. 28 (Diamantis), der ausdrücklich vom Vorwurf eines widersprüchlichen Verhaltens durch das vorlegende Gericht spricht.

87 EuGH v. 23.3.2000, Rs C-373/97, Slg. 2000, I-1705, Rz. 39 f. (Diamantis); in diesem Sinne auch bereits EuGH v. 12.3.1996 Rs. C-441/93, Slg. 1996, I-1347 Rz.70 (Pafitis) und EuGH v. 12.5.1998, Rs C-367/96, Slg. 1998, I-2843, Rz. 29 (Kefalas). 
wurde jedoch insoweit für möglich gehalten, als sich der Kläger verhältnismäßig spät auf die Nichtigkeit der Kapitalerhöhung berufen hat. ${ }^{88}$ Zwar könne es noch nicht für sich genommen mißbräuchlich sein, wenn innerhalb der nach nationalem Recht für Klagen der betreffenden Art geltenden Verjährungsfrist eine Klage, und sei es auch erst nach einer gewissen Zeit, erhoben werde. ${ }^{89}$ Etwas anderes könne jedoch gelten, wenn der Kläger unter den verschiedenen Rechtsbehelfen, die für die Behebung einer durch einen Verstoß gegen die Bestimmung des Art. 25 Abs. 1 entstandenen Lage zur Verfügung stehen, denjenigen ausgewählt hat, der den berechtigten Interessen Dritter einen derart schweren Schaden zufügt, daß er offensichtlich unverhältnismäßig ist. Eine solche Würdigung würde weder die Tragweite der genannten Bestimmung verändern noch ihre Zwecke beeinträchtigen. ${ }^{90}$ In der Entscheidung Diamantis läßt sich damit auch die Nähe des gemeinschaftsrechtlichen Rechtsmißbrauchsverbots zum Verhältnismäßigkeitsgrundsatz bzw. zum Übermaßverbot erkennen.

\section{Gleichbehandlungsgrundsatz}

Nach Art. 42 Kapital-RL müssen die „Rechtsvorschriften der Mitgliedstaaten die Gleichbehandlung der Aktionäre sicherstellen, die sich in denselben Verhältnissen befinden“. ${ }^{91}$ Aktionäre sollen danach auch aus Sicht des sekundären Gemeinschaftsrechts von der Gesellschaft unter gleichen Bedingungen gleich behandelt werden, sofern sie nicht einer abweichenden Behandlung zustimmen oder vorab durch eine Regelung im Gesellschaftsvertrag zugestimmt haben. Auch nach Art. 3 Abs. 1 lit. a Übernahme-RL sind alle Inhaber von Wertpapieren einer Zielgesellschaft, die der gleichen Gattung angehören, gleich zu behandeln. Art. 29 Abs. 1 Kapital-RL ordnet ein Bezugsrecht der Aktionäre im Verhältnis ihrer Anteile am Aktienkapital an. Bei der EWIV sehen Art.17 Abs.1 S. 1 bei der Stimmrechtsmacht und Art. 21 Abs. 1 und 2 EWIV-VO subsidiär bei der Gewinnund Verlustverteilung eine Gleichbehandlung nach Köpfen vor. Eine weitere Regelung zur Gleichbehandlung von Organmitgliedern enthält Art. 10a Abs. 1 des hier nicht näher zu behandelnden Kommissionsentwurfs für eine Struktur-RL. ${ }^{92}$ Das Gebot der Gleichbehandlung kann damit auch im Europäischen Gesellschaftsrecht bzw. im von ihm beeinflußten nationalen Gesellschaftsrecht als generalklauselartiger Maßstab zur Auslegung, Lückenfüllung und Anpassung des Gesellschaftsvertrags $^{93}$ sowie zur Kontrolle von Mehrheitsbeschlüssen herangezogen werden. ${ }^{94}$

Über die für seine Verallgemeinerungsfähigkeit bedeutsame Frage der Rechtfertigung des gesellschaftsrechtlichen Gleichbehandlungsgrundsatzes geben die gemeinschaftsrechtlichen Regelungen keinen näheren Aufschluß. Es bleibt offen, ob das Gleichbehandlungsgebot der Ausdruck einer allgemeinen Gerechtigkeitsidee ${ }^{95}$ bzw. der Treuepflicht ${ }^{96}$ ist oder ob es sich aus der Ausübung von organ- bzw. mitgliedschaftlichen Machtbefugnissen in der Gesellschaft ${ }^{97}$ oder aus dem durch die gemeinsame Zweckverfolgung entstehenden besonderen Gemeinschaftsverhältnis ergibt. ${ }^{98}$ Auch was das in letzter Zeit im Zusammenhang mit der Gleichbehandlungs-RL ${ }^{99}$ immer wieder heftig diskutierte Spannungsverhältnis zur Privatautonomie anbetrifft, kann dem Gesellschaftsrecht lediglich entnommen werden, daß der Gleichbehandlungsgrundsatz jedenfalls in Gemeinschaftsverhältnissen und auch dort nicht uneingeschränkt Geltung beanspruchen kann. Damit die Privatautonomie auch im Gemeinschaftsprivatrecht nicht zu sehr zurückgedrängt wird, sollte der Gleichbehandlungsgrundsatz außerhalb von besonderen Gemeinschaftsverhältnissen ${ }^{100}$ aber lediglich noch bei privater Machtausübung wie insbesondere aufgrund von Monopolstellungen zum Tragen kommen. ${ }^{101}$

\section{Verschuldensprinzip}

Das Verschulden bildet auch im Europäischen Gesellschaftsrecht eine grundsätzliche Voraussetzung der Schadensersatzhaftung von Organmitgliedern ${ }^{102}$ und Sachverständigen, ${ }^{103}$ deren Vorliegen teilweise vermutet wird. ${ }^{104}$ Eine ebenfalls verallgemeinerungsfähige Regelung stellt Art. 23 der Prüferbefähigungs-RL ${ }^{105}$ dar, wonach die Pflichtprüfer i.S.v. Art. 1 der RL von den Mitgliedstaaten zur Beachtung der „beruflichen Sorgfalt“ verpflichtet werden müssen. Hierbei handelt es sich um das Bekenntnis des Gemeinschaftsgesetzgebers zu einem objektiven und berufsspezifischen Sorgfaltsmaßstab. Es ist lediglich umstritten, ob es sich um einen europäisch autonom auszulegenden Begriff ${ }^{106}$ oder um einen nationalen Sorgfaltsstandard handelt. ${ }^{107}$

${ }^{88}$ Im Fall EuGH v. 23.3.2000, Rs C-373/97, Slg. 2000, I-1705 (Diamantis) betrug die Zeitspanne vier bzw. fünf Jahre.

${ }^{89}$ EuGH v. 23.3.2000, Rs C-373/97, Slg. 2000, I-1705, Rz. 39 (Diamantis).

90 EuGH v. 23.3.2000, Rs C-373/97, Slg. 2000, I-1705, Rz. 43 (Diamantis).

91 Siehe dazu Lutter, FS Ferid, 599, 605 ff. und Schön, RabelsZ 2000, 1, 25.

92 Siehe dazu Art.10 Abs. 1 der dritten Änderung des Vorschlags für eine Fünfte Richtlinie des Rates über die Struktur der Aktiengesellschaft sowie die Befugnisse und Verpflichtungen ihrer Organe v. 20.11.1991 KOM(91) 372 endg., ABl. C 321/9 v. 12.12.1991.

93 Vgl. dazu für das deutsche Recht L. Raiser, ZHR 111 (1948), 75, 80 und G. Hueck, Der Grundsatz der gleichmäßigen Behandlung im Privatrecht, 1958, S. $278 \mathrm{f}$.

94 Vgl. dazu für das deutsche Recht RG v. 15.5.1936 RGZ 151, 321, 326; BGH v. 14.5.1956 BGHZ 20, 363, 369; G. Hueck (Fn. 93), S. 41 und 308 ff.

95 So etwa L. Raiser, ZHR 111 (1948), 75, 90.

96 So etwa Fechner, Die Treubindungen der Aktionäre, 1942, S. 93 ff.; Hüffer, FS Steindorff, 1990, S. 59, 72; Lutter, JZ 1976, 225, 229; krit. L. Raiser, ZHR 111 (1948), 75, $83 \mathrm{f}$.

97 So etwa L. Raiser, ZHR 111 (1948), 75, 93; W. Böckenförde, Der alllgemeine Gleichheitssatz und die Aufgabe des Richters, 1957, S. 23 ff.; T. Raiser, Kapitalgesellschaften, § 12 Rn. 41 und K. Schmidt, Gesellschaftsrecht, § 16 II $4 \mathrm{~b}$.

98 So etwa G. Hueck (Fn. 93), S. 127 ff., 155 und 222 ff. und U. Huber, Vermögensanteil, Kapitalanteil, und Gesellschaftsanteil an Personengesellschaften des Handelsrechts, 1970, S. 314.

${ }^{99}$ RL 2000/43/EG des Rates v. 29.6.2000 zur Anwendung des Gleichbehandlungsgrundsatzes ohne Unterschied der Rasse oder der ethnischen Herkunft, ABl. L 180/22 v. 19.7.2000.

100 Beispiele bilden die Versichertengemeinschaft, die Gemeinschaft der Insolvenzgläubiger und die Betriebsgemeinschaft.

101 Beispiele bilden jedenfalls Art. 82 S. 2 lit. c EG und das Arbeitsverhältnis; siehe zum umstrittenen weiten Anwendungsbereich der Gleichbehandlungs-RL deren Art. 3.

102 Siehe dazu etwa Art. 20 Fusions-RL und Art. 18 Spaltungs-RL.

103 Siehe dazu etwa Art. 21 Fusions-RL und Art. 18 Spaltungs-RL.

104 Siehe dazu etwa Art. 18 Abs. 3 S. 2 Kapital-RL; für die Organmitglieder einer SE bzw. SCE mit Sitz in Deutschland gilt dies ebenfalls nach Art. 51 SE-VO i.V.m. § 93 Abs. 2 AktG bzw. Art. 51 SCE-VO i.V.m. § 34 Abs. 2 GenG.

105 RL 84/253/EWG des Rates v. 10.4.1984 über die Zulassung der mit der Pflichtprüfung der Rechnungslegungsunterlagen beauftragten Personen, ABl. L 126/20 v. 12.5.1984.

106 So etwa Grundmann, Europäisches Gesellschaftsrecht, 2004, Rn. 585.

107 So Ebke, ZVglRW 100 (2001), 62, 67 ff. 


\section{Notstandsprivileg}

Der allgemeine Gedanke des rechtfertigenden Notstands bzw. der Zulässigkeit der Notgeschäftsführung hat einen gesellschaftsrechtlichen Ausdruck in Art. 19 Abs. 2 Kapital-RL gefunden. Danach können die Mitgliedstaaten den ausnahmsweise zulässigen Erwerb eigener Aktien auch ohne Zustimmung der Hauptversammlung ermöglichen, wenn dies notwendig ist, um einen schweren unmittelbar bevorstehenden Schaden von der Gesellschaft abzuwenden.

\section{Beendigung von Dauerschuldverhältnissen aus wichtigem Grund}

Die allgemein im Privatrecht bestehende Möglichkeit zur Beendigung von Dauerschuldverhältnissen aus wichtigem Grund findet sich im Europäischen Gesellschaftsrecht in zweierlei Form. Zum einen kann der Gesellschafter einer EWIV die Gesellschaft nach Art. 27 Abs. 1 S. 2 EWIV-VO individuell durch eine Kündigung aus wichtigem Grund zur Auflösung bringen. Zum anderen steht den übrigen Gesellschaftern nach Art. 27 Abs. 2 EWIV-VO bzw. Art.15 Abs. 1 SCE-VO die Möglichkeit zum Ausschluß eines Gesellschafters und damit zur Beendigung des Mitgliedschaftsverhältnisses zur Verfügung, wenn der Gesellschafter grob bzw. schwerwiegend gegen seine Pflichten verstößt oder schwere Störungen der Arbeit der EWIV verursacht oder $\mathrm{zu}$ verursachen droht bzw. wenn er gegen die Interessen der SCE gehandelt hat.

\section{Bezugnahme des Europäischen Gesellschaftsrechts auf allgemein privatrechtliche Institute}

Eine größere Anzahl allgemein privatrechtlicher Institute wird im Europäischen Gesellschaftsrecht lediglich erwähnt und inhaltlich vorausgesetzt. Damit bleibt ihre nähere Ausgestaltung den nationalen Rechtsordnungen vorbehalten. Hier zeigt sich nicht nur die Einbettung des Europäischen Gesellschaftsrechts in das System des Gemeinschaftsprivatrechts, sondern auch seine Verknüpfung mit den mitgliedstaatlichen Privatrechten. Dies gilt zunächst für das Institut der unmittelbaren Stellvertretung mit den Unterformen der Gesamt- und Einzelvertretung ${ }^{108}$ und die Möglichkeit des Selbstkontrahierens ${ }^{109}$ sowie die mittelbare Stellvertretung..$^{100}$ Hierher gehören zudem die Rechtsinstitute der Schriftform, ${ }^{111}$ der öffentlichen Beurkundung, ${ }^{112}$ der Gesamtschuld, ${ }^{113}$ der Wahlschuld, ${ }^{114}$ des Erlasses, ${ }^{115}$ der Sicherheitsleistung, ${ }^{116}$ der vollständigen ${ }^{117}$ und partiellen ${ }^{118}$ Universalsukzession, der Vertragsnachfolge ${ }^{119}$ sowie der Enteignungsentschädigung. ${ }^{120}$ Interessanterweise kennt das Europäische Gesellschaftsrecht schließlich auch die relative und die vorübergehende Unwirksamkeit von Rechtsgeschäften. ${ }^{121}$

\section{Begriffsdefinitionen im Europäischen Gesellschaftsrecht von allgemein privatrechtlicher Bedeutung}

Da die meisten gesellschaftsrechtlichen Regelungen aus einer vergleichsweise frühen Phase der Gemeinschaftsrechtsetzung stammen, enthalten sie nur wenige für den jeweiligen Rechtsakt maßgebliche Begriffsdefinitionen, ${ }^{122}$ von denen noch weniger von allgemein privatrechtlichem Interesse sind. So wird etwa der Wertpapierbegriff in Art. 2 Abs. 1 lit. e Übernahme-RL letztlich nicht definiert, sondern vorausgesetzt und speziell im Hin- blick auf den Regelungsgegenstand der Übernahme-RL auf übertragbare Wertpapiere beschränkt, die Stimmrechte in einer Gesellschaft verleihen. Verallgemeinerungsfähig ist hingegen neben dem sogleich noch zu besprechenden Begriff der Rechtsfähigkeit die Definition der elektronischen Form i.S.v. Art. 3 der Publizitäts-RL durch Art. 3 Abs. 8 Publizitäts-RL, wonach diese vorliegt, wenn „die Information mittels Geräten für die elektronische Verarbeitung (einschließlich digitaler Kompression) und Speicherung von Daten am Ausgangspunkt gesendet und am

108 Siehe dazu etwa Art. 9 Abs. 3 Publizitäts-RL.

109 Art. 5 Einpersonen-GmbH-RL.

110 Siehe dazu etwa Art. 4 Abs. 12 SCE-V0 und zur Anerkennung der Einkaufskommission Art. 20 Abs. 1 lit. c Kapital-RL.

111 Siehe dazu etwa Art. 29 Abs. 3 S. 3 Kapital-RL, Art. 5 Abs. 1 Fusions-RL und Art. 5 Abs. 2 SCE-VO.

112 Siehe dazu etwa Art. 1 Publizitäts-RL und Art. 16 Abs. 1 Fusions-RL.

113 Siehe dazu Art. 22 Abs. 1 lit. h Fusions-RL (gesamtschuldnerische Haftung der an einer nichtigen Verschmelzung beteiligten Gesellschaften), Art. 12 Abs. 3 Spaltungs-RL (gesamtschuldnerische Ausfallhaftung der begünstigten Gesellschaften, wobei die Mitgliedstaaten einen Beschränkung auf das jeder dieser Gesellschaften zugeteilte Nettoaktivvermögen vorsehen können), Art. 12 Abs. 6 Spaltungs-RL (Möglichkeit der gesamtschuldnerischen Haftung der begünstigten Gesellschaften für die Verpflichtungen der gespaltenen Gesellschaft) sowie Art.7 Publizitäts-RL, Art. 9 Abs. 2 EWIV-VO, Art. 16 Abs. 2 SE-VO und Art. 17 Abs. 2 SCE-VO (unbeschränkte gesamtschuldnerische Handelndenhaftung).

114 Siehe dazu Art. 5 Abs. 5 S. 2 und 4 sowie Art. 15 Abs. 5 S. 3 ÜbernahmeRL.

115 Art. 12 Kapital-RL (Verbot des Erlasses von Einlageverpflichtungen).

116 Art. 32 Kapital-RL (Sicherheitsleistung bei Kapitalherabsetzung).

117 Art. 19 Abs. 1 lit. a Fusions-RL; Art. 29 Abs. 1 lit. a bzw. Abs. 2 lit. a SE-V0; siehe auch indirekt Art. 28 Abs. 2 EWIV-V0, der allerdings zugleich unter dem Gesichtspunkt „Gesellschaftsrecht geht vor Erbrecht“ eine Einschränkung der Universalsukzession von Todes wegen vorsieht. 118 Art. 17 Abs. 1 lit. a Spaltungs-RL.

119 Siehe dazu etwa Art. 29 Abs. 4 SE-V0 und Art. 35 Abs. 8 SCE-VO.

120 Siehe dazu Art. 11 Abs. 5 Übernahme-RL (angemessene Entschädigung der von den Durchbruchsregeln des Art.11 Abs. 2 bis 4 Übernahme-RL betroffenen Rechteinhaber für die diesen durch die Teilenteignung entstehenden Verluste).

121 Siehe dazu Art. 11 Abs. 2 Übernahme-RL (Beschränkungen in Bezug auf die Übertragung von Wertpapieren, die in der Satzung der Zielgesellschaft oder in nach Inkrafttreten der Übernahme-RL abgeschlossenen vertraglichen Vereinbarungen vorgesehen sind, gelten dem Bieter gegenüber während der in Art. 7 Abs. 1 festgelegten Frist für die Annahme des Angebots nicht), Art.11 Abs. 3 Übernahme-RL (Stimmrechtsbeschränkungen, die in der Satzung der Zielgesellschaft oder in nach Inkrafttreten der Übernahme-RL abgeschlossenen vertraglichen Vereinbarungen vorgesehen sind, entfalten in der Hauptversammlung, die gemäß Art. 9 über etwaige Abwehrmaßnahmen der Zielgesellschaft beschließt, keine Wirkung) und Art.11 Abs. 4 Übernahme-RL (vorübergehende Unwirksamkeit von Sonderrechten und Übertragungsbeschränkungen in der ersten Hauptversammlung nach Angebotsschluß).

122 Beispiele sind Art. 2 Abs. 2 Publizitäts-RL (Begriff der geschlossenen Aktiengesellschaft), Art. 1 Abs. 2 UAbs. 2 Kapital-RL und Art. 5 Abs. 2 Bilanz-RL (Begriff der Investmentgesellschaft), Art. 5 Abs. 3 Bilanz-RL (Begriff der Beteiligungsgesellschaft), Art. 17 Bilanz-RL (Begriff der Beteiligung), Art. 1 und 33 Abs. 1 S. 2 Konzernbilanz-RL (konzernrechtliche Begriffe) sowie Art. 2 der SE-RL 2001/86/EG v. 8.10.2001 ABl. L 294/22 v. 10.11.2001 (Umschreibung von Begriffen des kollektiven Arbeitsrechts). 
Endpunkt empfangen wird und sie vollständig über Draht, über Funk, auf optischem oder anderem elektromagnetischem Wege in der von den Mitgliedstaaten bestimmten Art und Weise gesendet, weitergeleitet und empfangen wird“. Von allgemeinem Interesse ist zudem die Definition des Begriffs „gemeinsam handelnde Personen" durch Art. 2 Abs. 1 lit. d Übernahme-RL. Hierbei handelt es sich um „natürliche oder juristische Personen, die mit dem Bieter oder der Zielgesellschaft auf der Grundlage einer ausdrücklichen oder stillschweigenden, mündlich oder schriftlich getroffenen Vereinbarung zusammenarbeiten, um die Kontrolle über die Zielgesellschaft zu erhalten bzw. den Erfolg des Übernahmeangebots zu vereiteln“.

\section{Privatrechtssystematische Unterscheidungen im Europäischen Gesellschaftsrecht}

Das Europäische Gesellschaftsrecht legt in seinen Regelungen zumeist stillschweigend einige wenige begriffliche Unterscheidungen zugrunde, denen eine privatrechtssystematische Bedeutung zukommt. Dies gilt zunächst für die Unterscheidung von Zivil- und Handelsrecht, wenn in Art. 48 Abs. 2 EG zwischen Gesellschaften bürgerlichen Rechts und Gesellschaften des Handelsrechts differenziert wird oder in Art. 1 Abs. 1 der SE-VO von „Handelsgesellschaften“"123 die Rede ist. In Art. 15 Abs. 1 Konzernbilanz-RL ${ }^{124}$ wird ferner in einer aus deutscher Sicht befremdlichen Weise zwischen gewerblicher Tätigkeit und Handeltreiben unterschieden, wobei beide Tätigkeiten zugleich von anderen Tätigkeiten abgegrenzt werden. Gleichfalls nur zum Teil stimmt die in Art. 4 lit. b EWIV-VO vorgenommene Unterscheidung zwischen gewerblicher, kaufmännischer, handwerklicher, landwirtschaftlicher und freiberuflicher Tätigkeit mit der deutschen Systematik überein. Da sich aus dieser Differenzierung jedoch keine Unterschiede in den Rechtsfolgen ergeben und der Verordnungsgeber vermutlich ${ }^{125}$ auch unter Inkaufnahme von Überschneidungen lediglich in Anlehnung an Art.50 EG alle denkbaren wirtschaftlichen Tätigkeiten erfassen wollte, sollte dieser Regelung keine begriffsbildende Kraft zugesprochen werden. Dem deutschen Juristen durchaus vertraut ist hingegen die vom EuGH vorgenommene Unterscheidung zwischen materieller Handelsregisterpublizität und allgemeiner Rechtsscheinhaftung. ${ }^{126}$

Bei den Rechtssubjekten wird generell zwischen natürlichen und juristischen Personen ${ }^{127}$ sowie teilweise - offenbar als $\mathrm{Zu}$ geständnis etwa an die deutsche Dogmatik - auch noch sonstigen Gesellschaften ${ }^{128}$ unterschieden. Die juristischen Personen werden zudem in solche des öffentlichen Rechts und des privaten Rechts geschieden. ${ }^{129}$ Interessanterweise findet sich in Art. 1 Abs. 2 und 3 EWIV-VO auch die aktuell im deutschen Recht vorherrschende, ${ }^{130}$ aber in vielen anderen Rechtsordnungen unbekannte Unterscheidung zwischen Rechtsfähigkeit und Rechtspersönlichkeit. Während die Rechtsfähigkeit als Fähigkeit umschrieben wird, ,im eigenen Namen Träger von Rechten und Pflichten jeder Art zu sein, Verträge zu schließen oder andere Rechtshandlungen vorzunehmen und vor Gericht $\mathrm{zu}$ stehen“, wird die Verleihung der Rechtspersönlichkeit an die EWIV den Mitgliedstaaten freigestellt, ohne daß dabei allerdings wie auch in Art. 1 Abs. 3 SE-VO oder Art. 1 Abs. 5 SCE-VO näher auf den Inhalt der Rechtspersönlichkeit und den Unterschied zur Rechtsfähigkeit eingegangen werden würde. Während die Rechtsfähigkeit i.S.v. Art. 1 Abs. 2 EWIV-VO auch die Fähigkeit umfaßt, vor Gericht zu stehen, wird im Europäischen Gesellschaftsrecht ansonsten durchaus zwischen Rechtsfähig- keit und Parteifähigkeit bzw. zwischen materiellrechtlichen und prozeßrechtlichen Handlungen unterschieden. ${ }^{131}$

Aus Art. 7 S. 2 Kapital-RL bzw. Art. 18 Abs. 1 und 2 KapitalRL folgt schließlich noch, daß auch das Europäische Gesellschaftsrecht zwischen Arbeitsleistungen und (freien) Dienstleistungen bzw. zwischen unmittelbarer und mittelbarer Stellvertretung $^{132}$ unterscheidet.

\section{Beiträge des Europäischen Gesellschaftsrechts zu allgemeinen Fragen der Gemeinschaftsrechtsetzung}

\section{Nutzung von Selbstregulierungsmechanismen}

Was die Rechtsquellen des Gemeinschaftsprivatrechts anbetrifft, so kann gerade im Gesellschaftsrecht die Tendenz zur Integration informeller Regelungen festgestellt werden. Zwar kennt das Europäische Gesellschaftsrecht keinen eigenen Corporate Governance-Kodex und wird auf absehbare Zeit auch über keinen derartigen Kodex verfügen. ${ }^{133}$ Die Kommission strebt jedoch eine Koordinierung der in den Rechtsordnungen der Mitgliedstaaten ${ }^{134}$ bereits weit verbreiteten Corporate Go-

123 Dies gilt allerdings, soweit ersichtlich, nur für die deutsche Fassung.

124 RL 83/349/EWG des Rates v. 13.6.1983 über den konsolidierten Abschluß, ABl. L 193/1 v. 18.7.1983, zuletzt geändert durch RL 2003/51/EG des Europäischen Parlaments und des Rates v. 18.6.2003 zur Änderung der Richtlinien 78/660/EWG, 83/349/EWG und 91/674/EWG über den Jahresabschluß und den konsolidierten Abschluß von Gesellschaften bestimmter Rechtsformen, von Banken und anderen Finanzinstituten sowie von Versicherungsunternehmen, ABl. 2003 L 178/16 v. 17.7.2003.

125 Vgl. dazu den Erwägungsgründe 5 und 6 der EWIV-VO, wonach „der Begriff der wirtschaftlichen Tätigkeit im weitesten Sinne auszulegen“ ist und natürlichen Personen der Zugang zur EWIV „so weit wie möglich“ eröffnet werden soll.

126 Siehe dazu EuGH v. 20.9.1988, Rs.C-136/87, Slg. 1988, 4665, 4682, Rz. 13 ff. (Ubbink Isolatie BV/Dak -en Wandtechniek BV).

127 Eine Zweiteilung der Rechtssubjekte findet sich etwa in Art. 2 Abs. 1 lit. c und Art. 5 Abs. 1 Übernahme-RL sowie in Art. 14 Abs. 1 UAbs. 4 SCEVO.

128 Eine Dreiteilung der Rechtssubjekte findet sich etwa in Art. 3 lit. i, Art. 10 Abs. 1 S. 2 und Art. 27 Abs. 2 S. 2 Kapital-RL sowie in Art. 10 Abs. 1 S. 3 Fusions-RL und Art. 1 Abs. 2 Prüferbefähigungs-RL.

129 Siehe dazu etwa Art. 48 Abs. 2 EG.

130 Siehe zur Differenzierung zwischen Rechtsfähigkeit und Rechtspersönlichkeit im deutschen Recht die §§ 14, 21 f., 54, 1059 BGB und § 11 InsO sowie etwa $U$. Huber, FS Lutter, 2000, S. 107, 113 f. und Ulmer, ZIP 2001, 585, 588.

131 Siehe dazu etwa Art. 2 Abs. 1 lit. d i) Publizitäts-RL und EuGH v. 5.11.2002 Rs. C-208/00, Slg. 002, I-9919, Rz. 93 (Überseering).

132 Vgl. demgegenüber zur Agency im Common Law nur Zweigert/Kötz, Einführung in die Rechsvergleichung, 3. Aufl. 1996, S. 429 f.

133 Siehe dazu die ablehnende Stellungnahme in Europäische Kommission, Mitteilung an den Rat und das Europäische Parlament „Modernisierung des Gesellschaftsrechts und Verbesserung der Corporate Governance in der Europäischen Union - Aktionsplan“ v. 21.5.2003 KOM(2003) 284 endg., S. 12 ff.; zur Entwicklungsgeschichte etwa Haak, in: Gesellschaftsrechtliche Vereinigung (Hrsg.), Gesellschaftsrecht in der Diskussion 2003, S. 151, $152 \mathrm{ff}$.

134 Siehe dazu die von der Kommission in Auftrag gegebene ausführliche Studie von Weil, Gotshal \& Manges, Comparative Study of the Corporate Governance Codes relevant to the European Union and its Member States, http://europa.eu.int/comm/internal_market/en/company/com- 
vernance-Kodizes an. ${ }^{135}$ Damit macht sich auch die Europäische Gemeinschaft das Prinzip der Selbstverpflichtung von börsennotierten Gesellschaften zu eigen, die auf der Suche nach günstigen Finanzierungsmöglichkeiten mehr oder weniger freiwillig bereit sind, über die gesetzlichen Regelungen hinausgehende und das Vertrauen der Anleger fördernde Anforderungen zu erfüllen. ${ }^{136}$ Die mit Hilfe einer Richtlinie geplante Koordinierung wird dabei die schon jetzt etwa im deutschen Recht zu verzeichnende staatliche Einflußnahme auf den Kodexinhalt verstärken und die Regelungen damit noch mehr in die Nähe einer staatlichen Maßnahme rücken. ${ }^{137}$

Dem Prinzip der Selbstverpflichtung zeigt sich das Europäische Gesellschaftsrecht auch insoweit aufgeschlossen, als es etwa in Art. 1 Abs. 1 Übernahme-RL die verschiedenen unverbindlichen nationalen Kodizes zum Verhalten bei Übernahmen neben den Rechts- und Verwaltungsvorschriften sowie sonstigen Regelungen der Mitgliedstaaten ausdrücklich zu den „Vorschriften“ für Übernahmeangebote zählt, deren Koordinierung die Richtlinie anstrebt. Der Erhalt der bisherigen Beschäftigungsbedingungen bei Verschmelzungen nach Art. 29 Abs. 4 SE-VO und Art. 35 Abs. 8 SCE-VO umfaßt schließlich neben den einzelstaatlichen Rechtsvorschriften und individualvertraglichen Bedingungen auch sog. „Gepflogenheiten“, ${ }^{138}$ zu denen man neben dem Gewohnheits- und Richterrecht auch die betriebliche Übung zählen kann.

Ein gesellschaftsrechtliches Beispiel für die gleichfalls im Vordringen begriffene Verlagerung von Regelungszuständigkeiten auf demokratisch nicht legitimierte private Gremien bildet die IFRS-Verordnung. ${ }^{139}$ Auf ihrer Grundlage hat sich die EU-Kommission inzwischen für die International Financial Reporting Standards (IFRS) des International Accounting Standards Committee (IASC) als Internationale Rechnungslegungsstandards entschieden. ${ }^{140}$ Von besonderem Interesse ist dabei, daß die IFRS-Verordnung in Art. 3 Abs. 2 Kriterien wie etwa die Vereinbarkeit mit dem true and fair view-Prinzip benennt, die die IFRS als Voraussetzung für ihre Wahl durch die Europäische Kommission und sicherlich auch ihre fortbestehende Maßgeblichkeit erfüllen müssen. Mit diesem Modell könnte auch in anderen vergleichbaren Fällen die Vereinbarkeit mit deutschen ${ }^{141}$ und europäischen ${ }^{142}$ verfassungsrechtlichen Vorgaben nach Art des deutschen Wesentlichkeitsgrundsatzes hergestellt werden.

\section{Bedingte und fakultative Geltung von Gemeinschaftsrecht}

Als Vorreiter erweist sich das Gesellschaftsrecht schließlich auch hinsichtlich der bedingten und fakultativen Geltung von gemeinschaftsprivatrechtlichen Regelungen. Bei der hier als bedingt bezeichneten Geltung ist die nationale Maßgeblichkeit der Regelung von der Existenz eines entsprechenden Rechtsinstituts im jeweiligen Mitgliedstaat abhängig. Dies gilt etwa für sämtliche Regelungen zur Spaltung (Art.1 Spaltungs-RL) und die Regelungen der Einpersonen-GmbH-RL zur Einpersonen-AG (Art. 6 Einpersonen-GmbH-RL). Von einer fakultativen Geltung kann man demgegenüber sprechen, wenn die Anwendbarkeit ganzer Rechtsakte oder maßgeblicher Bestandteile derselben unter den Vorbehalt ihrer Annahme durch die Mitgliedstaaten bzw. einzelne Privatrechtssubjekte gestellt wird. ${ }^{143}$ Ein zuletzt heftig diskutiertes Beispiel bildet Art. 12 Übernahme-RL, der die zentralen Durchbruchsregelungen der Richtlinie für die Mitgliedstaaten nur insoweit für verbindlich erklärt, als diese den Gesellschaften i.S.d. Art. 1 Abs. 1 Übernahme-RL die
Möglichkeit zu einer widerruflichen und gegebenenfalls nach Art. 12 Abs. 3 Übernahme-RL auszusetzenden Wahl zugunsten dieser Durchbruchsregelungen einzuräumen haben. Die Mitgliedstaaten erhalten mithin das Recht zum opt out unter der Voraussetzung, daß sie diese Abwahl nicht zwingend ausgestalten und den ihrem Gesellschaftsstatut sowie der Richtlinie unterworfenen Gesellschaften das Recht zum opt in gewähren. Zwar handelt es sich hierbei um eine dem politischen Kompromiß geschuldete Konstruktion, die im Hinblick auf das angestrebte Ziel einer Harmonisierung des Übernahmerechts mit Recht nicht nur von der Europäischen Kommission heftig kritisiert wurde. ${ }^{144}$ Gerade vor dem Hintergrund der hier interessierenden Fragen ist aber auch hervorzuheben, daß es sich in zweierlei Hinsicht durchaus um einen zukunftsweisenden Ansatz handelt. Zum einen wird nämlich der Umstand, ob eine Gesellschaft aufgrund gesetzlicher Vorgabe bzw. eigener Wahl letztlich dem gesamten gemeinschaftsrechtlichen Übernahmeregime untersteht oder nicht, von den Kapitalmärkten bewertet und damit beeinflußt werden. Zum anderen wird hier die Möglichkeit $\mathrm{zu}$ einer differenzierten Integration ${ }^{145}$ im Gemeinschaftsprivatrecht geschaffen, deren konkrete Ausgestaltung nicht nur durch Entscheidungen von Mitgliedstaaten, sondern auch von Privatrechtssubjekten beeinflußt wird.

pany/news/corp-gov-codesrpt_en.htm sowie den analytischen Überblick von R. Schmidt, in: Berndt (Hrsg.), Leadership in turbulenten Zeiten, 2003, S. 71 ff. und näher Hopt (Hrsg), Comparative Corporate Governance - The State of the Art and Emerging Research, 1998.

135 Europäische Kommission, Mitteilung an den Rat und das Europäische Parlament „Modernisierung des Gesellschaftsrechts und Verbesserung der Corporate Governance in der Europäischen Union - Aktionsplan“ v. 21.5.2003 KOM(2003) 284 endg., S. 14 ff.

136 Dazu auch Wymeersch, ZGR 2001, 294, 314; krit. im Hinblick auf die Freiwilligkeit der Selbstverpflichtung gerade auch betroffener Dritter und die fehlende gesetzgeberische Legitimation der Kodexregelungen M. Wolf, ZRP 2002, 59, 60 und Seidel, ZIP 2004, 285, 290 ff.

137 Siehe zur Qualifikation des Deutschen Corporate Governance-Kodexes als einer staatlichen Regelung bereits Seidel, ZIP 2004, $285 \mathrm{ff}$.

138 Auch in der RL 2001/86/EG v. 8.10.2001 zur Ergänzung des SE-Statuts hinsichtlich der Beteiligung der Arbeitnehmer, ABl. L 294/22 v. 10.11.2001, findet sich passim die Unterscheidung von Rechtsvorschriften und Gepflogenheiten.

139 VOEG/1606/2002 v. 19.7.2002 betreffend die Anwendung internationaler Rechnungslegungsstandards, ABl. 2002 L 243/1 v. 11.9.2002.

140 VO EG/1725/2003 der Kommission v. 29.9.2003 betreffend die Übernahme bestimmter internationaler Rechnungslegungsstandards in Übereinstimmung mit der VO EG/1606/2002 des Europäischen Parlaments und des Rates, ABl. L 261/1 v. 13.10.2003, geändert durch VO EG/707/2004 der Kommission vom 6.4.2004 zur Änderung der VO EG/1725/2003 ABl. L 111/3 v. 17.4.2004.

141 Siehe dazu eingehend im Zusammenhang mit der (seinerzeit noch geplanten) Regelung des § 292a HGB Hommelhoff, FS Odersky, 1996, $779 \mathrm{ff}$.

142 Dazu näher Heintzen, BB 2001, 825 ff.

143 Siehe auch die Diskussion um Optionsmodelle im europäischen Vertragsrecht (dazu nur Basedow, ZEuP 2004, 1 ff.; Schmidt-Kessel/Najork, GPR 2003/04, 5, 10 f. und Zypries, ZEuP 2004, 225, 230 f.).

144 Siehe etwa Germain, GPR 2003/04, 150, $151 \mathrm{f}$.

${ }_{145} \mathrm{Zu}$ den Problemen einer differenzierten Integration im Gemeinschaftsprivatrecht siehe den Überblick bei Jung, GPR 2003/04, 169. 


\section{Sprachenfrage}

Sprachregelungen haben eine besondere Bedeutung im Recht der möglichst gemeinschaftsweit herzustellenden Unternehmenspublizität und sind dort auch besonders umstritten. Die Gemeinschaft hat sich hier auf eine minimale Kompromißlösung zurückgezogen, indem sie es den Mitgliedstaaten überläßt, die Sprache(n) der obligatorischen Offenlegung zu bestimmen. ${ }^{146}$ Daneben wird den Mitgliedstaaten aber auch die Möglichkeit eröffnet, weitere Amtssprachen ${ }^{147}$ oder auch andere Sprachen ${ }^{148}$ für eine fakultative Publizität zuzulassen. Nach Art. 3a Abs. 2 Publizitäts-RL sind die Mitgliedstaaten sogar zur Zulassung jeder anderen Amtssprache für die fakultative Offenlegung verpflichtet. Im Falle von Abweichungen können die freiwillig offen gelegten Übersetzungen Dritten zwar nicht entgegengehalten werden, diese haben jedoch die Möglichkeit, sich bei Unkenntnis der obligatorischen Sprachfassung auf die fakultativ offen gelegten Übersetzungen zu berufen. ${ }^{149}$ Auch nach Art. 19 der Prospekt$\mathrm{RL}^{150}$ ist zunächst die Sprache des Herkunftsstaats für den Gesamtprospekt maßgeblich. Sofern jedoch allein oder auch in anderen Ländern als dem Herkunftsstaat Märkte betroffen sind, kann jeder betroffene Staat eine Übersetzung der Zusammenfassung in seine Amtssprache verlangen.

\section{Höchst-und Mindeststandard}

Die verschiedentlich heftig diskutierte Frage, ob die jeweilige Richtlinie nur einen Mindeststandard oder auch einen Höchststandard festlegt, hat im Europäischen Gesellschaftsrecht keine generelle, sondern eine wie auch sonst am jeweiligen Wortlaut und Regelungszweck sowie den primärrechtlichen Vorgaben orientierte differenzierte Lösung erfahren. ${ }^{151}$ Nur in einigen Fällen wurde die Frage ausdrücklich geregelt. ${ }^{152}$ Was das Bezugsrecht nach Art. 29 Abs. 4 Kapital-RL anbetrifft, so hat sich der EuGH für eine Mindestregelung ausgesprochen. ${ }^{153}$ Art. 2 Abs. 2 Zweigniederlassungs-RL ${ }^{154}$ hält er hingegen für eine Höchstregelung. ${ }^{155}$ Noch nicht endgültig geklärt ist die Natur der in Art. 11 Abs. 1 Kapital-RL enthaltenen Nachgründungsregelung und damit die Möglichkeit zu einem weitergehenden nationalen Sachgründungsrecht. ${ }^{156}$

\section{Verweisungs- und Vorrangregelungen}

Die Richtlinien und Verordnungen des Europäischen Gesellschaftsrechts enthalten zahlreiche Verweisungen, ${ }^{157}$ wobei die Terminologie für die besonderen Zwecke der jeweiligen Regelung gegebenenfalls angepaßt wird. ${ }^{158}$ Wenn die Bestimmungen der Bilanz-RL ${ }^{159}$ gemäß deren Art. 2 Abs. 5 gegebenenfalls auch gegen ihren Wortlaut so anzuwenden sind, daß dem true and fair view-Prinzip entsprochen wird, so wird dieses nicht nur zum overriding principle erklärt, sondern auch der Vorrang der teleologischen vor der grammatischen Auslegung festgeschrieben. Aus der in Art. 28 Abs. 2 EWIV-Vo getroffenen Regelung läßt sich zudem der allgemeine Grundsatz des Vorrangs des Personengesellschaftsrechts vor dem Erbrecht entnehmen.

\section{Monitoring}

Schon verhältnismäßig frühzeitig hat der Gemeinschaftsgesetzgeber im Gesellschaftsrecht schließlich damit begonnen, Regelungen zur Überwachung der gleichmäßigen Anwendung bzw. Durchführung von Richtlinien und Verordnungen durch Kon- taktausschüsse aus Vertretern der Mitgliedstaaten und der Kommission zu schaffen. ${ }^{160}$ Die Kommission hat zudem vielfach auch ausdrücklich die Aufgabe erhalten, die getroffenen Regelungen auf ihre Reformbedürftigkeit hin zu überprüfen, ${ }^{161}$ wobei sie von den Kontaktausschüssen ${ }^{162}$ bzw. dem Europäischen Wertpapierausschuß $\beta^{163}$ unterstützt werden soll. Von Interesse für die allgemeine Rechtsquellenlehre ist schließlich noch Art. 18 Abs. 3 Übernahme-RL, der als eine Art Sunset-Rule nach vier Jahren die Aussetzung der gemäß Art. 18 Abs. 2 Übernahme-RL im Ausschußverfahren erlassenen Durchführungsmaßnahmen vorsieht, wobei nach Überprüfung eine Verlängerungsentscheidung im Mitentscheidungsverfahren möglich ist.

146 Art. 3a Abs. 1 Publizitäts-RL.

147 So in Art. 4 Zweigniederlassungs-RL.

148 So in Art. 3a Abs. 3 Publizitäts-RL.

149 Siehe dazu auch Schemmann, GPR 2003/04, 92, $93 \mathrm{f}$.

150 RL 2003/71/EG des Europäischen Parlaments und des Rates v. 4.11.2003 betreffend den Prospekt, der beim öffentlichen Angebot von Wertpapieren oder bei deren Zulassung zum Handel zu veröffentlichen ist, und zur Änderung der Richtlinie 2001/34/EG, ABl. L 345/64 v. 31.12.2003.

151 Dazu näher Steindorff, EuZW 1990, 251 ff.; siehe auch Drygala, AG 2001, 291, $293 \mathrm{ff}$.

152 Siehe etwa Art. 2 und 3 Kapital-RL und Art. 3 Abs. 2 lit. b Übernahme-RL einerseits (Mindestregelung) sowie Art.17 Kapital-RL und Art. 2 f. Zweigniederlassungs-RL andererseits (Höchstregelung).

153 EuGH v. 19.11.1996 Rs. C-42/95, Slg.1996, I-6017, Rz. 12 ff. (Siemens/ Nold).

154 RL 89/666/EWG des Rates v. 21.12.1989 über die Offenlegung von Zweigniederlassungen, die in einem Mitgliedstaat von Gesellschaften bestimmter Rechtsform errichtet wurden, die dem Recht eines anderen Staates unterliegen, ABl. L 395/36 v. 30.12.1989.

155 EuGH v. 30.9.2003 Rs.C-167/01 (Inspire Art), NJW 2003, $3331=$ AG 2003, 680 .

156 Siehe dazu den Fall EuGH v. 16.7.1992, Rs. C-83/91, Slg. 1992, I-4919 Rz. 25 ff. (Meilicke) sowie Einsele, NJW 1996, 2681, 2683 f.

157 Beispiele finden sich insbesondere in der Spaltungs-RL, der Konzernbilanz-RL und der Zweigniederlassungs-RL.

158 Siehe z.B. Art. 2 Abs. 3 Spaltungs-RL.

159 RL 78/660/EWG des Rates v. 25.7.1978 über den Jahresabschluß von Gesellschaften bestimmter Rechtsformen, ABl. L 222/11 v. 14.8.1978, zuletzt geändert durch RL 2003/51/EG des Europäischen Parlaments und des Rates v. 18.6.2003 zur Änderung der Richtlinien 78/660/EWG, 83/349/EWG und 91/674/EWG über den Jahresabschluß und den konsolidierten Abschluß von Gesellschaften bestimmter Rechtsformen, von Banken und anderen Finanzinstituten sowie von Versicherungsunternehmen, ABl. 2003 L 178/16 v. 17.7.2003.

160 Siehe dazu Art. 52 Abs. 1 lit. a Bilanz-RL, Art. 29 lit. a Prüferbefähigungs-RL, Art. 17 lit. a Zweigniederlassungs-RL und Art. 42 Abs. 1 lit. a EWIV-VO.

161 Siehe dazu etwa Art. 6 Abs. 3 Kapital-RL, Art. 53 Abs. 2 Bilanz-RL; Art. 50 Abs. 1 Konzernbilanz-RL, Art. 20 Übernahme-RL und Art. 69 SEVo.

162 Siehe dazu Art. 52 Abs. 1 lit. b Bilanz-RL, Art. 29 lit. b Prüferbefähigungs-RL, Art. 17 lit. b Zweigniederlassungs-RL und Art. 42 Abs. 1 lit. b EWIV-VO.

163 Siehe dazu Art. 18 Abs. 1 Übernahme-RL. 


\section{Fazit}

Die vorstehenden Ausführungen haben verdeutlicht, daß das Europäische Gesellschaftsrecht zahlreiche allgemein privatrechtliche Regelungen und Rechtsinstitute kennt, die vielfach auch als systembildend für das Gemeinschaftsprivatrecht insgesamt bezeichnet werden können. Bei der Lösung von allgemeinen Fragen der Gemeinschaftsrechtsetzung erweist sich das Gesellschaftsrecht sogar durchaus als innovativ. Dennoch bleibt das Gesellschaftsrecht insoweit hinter den (vielleicht auch zu hohen) Erwartungen zurück, die es als historisch erstes und nach wie vor zentrales Gebiet der europäischen Rechtsangleichung weckt. Die Gründe hierfür sind vielfältig: In der Natur des Gesellschaftsrechts als einer Sondermaterie des Privatrechts und Teilmaterie des Unternehmensrechts liegt es zunächst begründet, daß die in diesem Bereich besonders weitreichenden Regelungen zur Publizität und zum Verkehrsschutz nur eingeschränkt verallgemeinert werden können. Ein Rechtsinstitut wie die mitgliedschaftliche Treuepflicht ist zwar Ausdruck eines systembildenden Rechtsprinzips, kann jedoch nur in Personenverbänden und damit im Gesellschaftsrecht zum Tragen kommen. Auch der im Bereich spezifisch gesellschaftsrechtlicher Kapitalgesellschafts- und Rechnungslegungsregelungen liegende Schwerpunkt der Rechtsetzung trägt zu ihrer verhältnismäßig geringen Verallgemeinerungsfähigkeit bei. Einen weiteren Grund bildet die Tatsache, daß der überwiegende Teil des geltenden Europäischen Gesellschaftsrechts einer vergleichsweise frühen Phase des Gemeinschaftsprivatrechts entstammt. So ist im Gesellschaftsrecht die inzwischen abnehmende Zurückhaltung des Gesetzgebers und des $\mathrm{EuGH}^{164}$ gegenüber umfassenden und in die mitgliedstaatlichen Privatrechtssysteme eingreifenden Regelungen noch deutlich spürbar. Die Richtlinien und Verordnungen enthalten darüber hinaus keine oder nur wenige Begriffsdefinitionen, die zudem nur selten allgemein privatrechtliche Bedeutung haben oder gar auf eine Systembildung hin angelegt wären. Nicht selten wurden schließlich ursprünglich noch wesentlich umfassendere Rechtsetzungsvorhaben zusammengestrichen, um sie insbesondere in ihrem gesellschaftsrechtlichen Kernanliegen politisch überhaupt durchsetzen zu können. ${ }^{165}$ Mit Spannung bleibt jedoch zu beobachten, welche Auswirkungen die weiteren gesellschaftsrechtlichen Vorhaben der Europäischen Kommission auf das Gemeinschaftsprivatrecht haben werden.

\section{Summary}

The preceding remarks point out that European company law consists of many regulations relating to general aspects of private law. Nevertheless, company law as a central element in the unifying of European law does not meet the expectations in all cases. The reasons for this are manifold: it's in the nature of company law that regulations on publicity and traffic protection are strictly limited to this field of law and can not be generalized. This is also true in the central matters of accounting and capital protection. Additionally, in this field, the legislator and the Court of Justice are reluctant towards extensive regulations that conflict with the private law systems of the Member States.

\section{Résumé}

Cet article présente les éléments généraux de droit privé dans le droit communautaire des sociétés actuellement en vigueur et tend à vérifier l'impact de ces éléments sur le système du droit privé communautaire dans son ensemble. Il montre que le droit européen des sociétés en tant que matière fondamentale du droit privé communautaire influence considérablement le système de ce dernier, bien que, pour des multiples raisons, l'impact soit moins important qu'on pourrait le penser.

164 Zur Selbstbeschränkung des EuGH im Bereich des Gesellschaftsrechts Everling, FS Lutter, 2000, S. 31, 43 f.

165 So wurden beispielsweise die Regelungen zur Organhaftung des Art. 77 SE-VOV 1991 durch einen schlichten Verweis auf das anwendbare nationale Recht in Art. 51 SE-VO ersetzt. 